\title{
Controllability and motion planning of a multibody Chaplygin's sphere and Chaplygin's top
}

\author{
Jinglai Shen ${ }^{1, * \dagger}$, David A. Schneider ${ }^{2}$ and Anthony M. Bloch ${ }^{2}$ \\ ${ }^{1}$ Department of Mathematics and Statistics, University of Maryland Baltimore County, \\ Baltimore, MD 21250, U.S.A. \\ 2 Department of Mathematics, University of Michigan, Ann Arbor, MI 48109 U.S.A.
}

\begin{abstract}
SUMMARY
This paper studies local configuration controllability of multibody systems with nonholonomic constraints. As a nontrivial example of the theory, we consider the dynamics and control of a multibody spherical robot. Internal rotors and sliders are used as the mechanisms for control. Our model is based on equations developed by the second author for certain mechanical systems with nonholonomic constraints, e.g. Chaplygin's sphere and Chaplygin's top in particular, and the multibody framework for unconstrained mechanical systems developed by the first and third authors. Recent methods for determining controllability and path planning for multibody systems with symmetry are extended to treat a class of mechanical systems with nonholonomic constraints. Specific results on the controllability and path planning of the spherical robot model are presented. Copyright (c) 2007 John Wiley \& Sons, Ltd.
\end{abstract}

Received 16 February 2007; Revised 23 May 2007; Accepted 23 June 2007

KEY WORDS: nonholonomic systems; controllability; motion planning

\section{INTRODUCTION}

The classical theory of rolling rigid bodies has a rich history. Vector-based formulations, as opposed to local coordinates on $S O(3)$, date back to Routh [1] and Chaplygin (translated in [2]). Recent work includes [3-6]. Control of a rolling ball has also received considerable interest in the control and robotics community. The plate-ball problem, a kinematic control problem where the horizontal components of the ball's spatial angular velocity are controlled, is introduced in [7]. Explicit solutions to the plate-ball problem are found in $[8,9]$. The paper [10] deals with the

\footnotetext{
*Correspondence to: Jinglai Shen, Department of Mathematics and Statistics, University of Maryland Baltimore County, Baltimore, MD 21250, U.S.A.

${ }^{\dagger}$ E-mail: shenj@umbc.edu

Contract/grant sponsor: NSF; contract/grant numbers: DMS-0305837, DMS-0606307, CMS-0408542

Contract/grant sponsor: Mittag Leffler Institute
}

Copyright (c) 2007 John Wiley \& Sons, Ltd. 
motion planning problem of a sphere controlled via rotors or sliders: it is concerned firstly with the application of differential geometric tools to controllability analysis, and secondly with motion planning via series approximation and perturbations.

Controllability is a fundamental issue in systems and control theory. In addition to its theoretical importance, it is also closely related to control design such as motion planning scheme development. Controllability analysis of nonlinear systems relies heavily on computing Lie brackets and determining the rank of the accessibility distribution [11]. This task quickly becomes intractable for many systems. Various tools from geometric mechanics and nonlinear control theory have been successfully applied to mechanical systems, e.g. [12-14]. The paper [13] defines the notions of local configuration accessibility and equilibrium controllability, and provides general sufficient controllability conditions for simple mechanical systems. It also shows that determining the rank of the accessibility distribution at an equilibrium can greatly simplify the calculations; see a brief review in Section 2 of the present paper. However, carrying out these calculations in generalized coordinates for multibody systems on a manifold such as $S O(3)$ would still be complicated. Methods for evaluating local configuration controllability of multibody systems with symmetry are developed in [15]. See [14] for a general theory of configuration controllability for simple mechanical systems with constraints.

The present paper, motivated by the dynamics and control of a spherical robot, is concerned with local configuration controllability and path planning for a class of multibody systems with nonholonomic constraints controlled via internal actuators. The dynamics and constraints treated in this paper can be formulated in terms of an advected parameter, and lead to Euler-Poincaré equations on the reduced space. The study of this class of systems is based on the results of [6] for certain nonholonomic systems and the multibody and control framework for unconstrained systems developed in [15]. The control analysis and design tools of [15] are extended to multibody systems with symmetry and nonholonomic constraints by using the results of [6]. As a nontrivial example of the theory, we consider the dynamics and control of a multibody spherical robot proposed by [10]. This robot can be treated in two interesting but rather different cases: a controlled Chaplygin's sphere and a controlled Chaplygin's top. The sphere has a nonhomogeneous mass distribution but with its center of mass located at the geometric center of the ball. The top, on the other hand, does not have its center of mass at the geometric center. Internal rotors and then sliders are considered as the mechanism for control. First, local configuration/equilibrium controllability as defined in [13] is investigated. We find that generically, both the two- and three-rotor cases are locally configuration/equilibrium controllable at equilibria, when gravity does not affect the system. We also give sufficient controllability conditions for the rotor cases when gravitational effects exist. A study of the slider case, where Lie brackets up to second order are computed, produces conditions that will generically not be satisfied, suggesting it is a more difficult model to control. We next consider motion planning for the three-rotor cases, and obtain controls required to produce both a pure translation and a pure rotation of the spherical body. Partial results of this work can be found in [16].

The rest of the paper is organized as follows. Section 2 reviews background information on simple mechanical control systems, symmetric products, and related controllability notions. In Section 3, we introduce a class of multibody systems with nonholonomic constraints that fit the framework of Euler-Poincare equations and describe the spherical robot model as an example. We then present in Section 4 the computation of Lie brackets and symmetric products within this framework and sufficient conditions for controllability. Section 5 focuses on controllability analysis of the spherical robot, with the ball center of mass at or off the geometric center, which 
corresponds to a controlled Chaplygin's sphere or top, respectively; the detailed controllability results are given for various cases. Finally, we consider motion planning for Chaplygin's sphere and top with three rotors in Section 6.

\section{BACKGROUND}

We briefly present the basic setting and notions for local controllability of simple mechanical systems, following $[13,14]$. Sufficient controllability conditions in terms of symmetric products and Lie brackets are summarized. Additional controllability results are available in [17] for constrained and unconstrained simple mechanical systems with symmetry but without potential.

\subsection{Simple mechanical control systems and symmetric products}

Consider a simple mechanical system with the following Lagrangian:

$$
L(q, \dot{q})=\frac{1}{2} \sum_{i, j=1}^{n} g_{i j}(q) \dot{q}^{i} \dot{q}^{j}-V(q)
$$

where $q=\left(q_{1}, \ldots, q_{n}\right)$ are local coordinates of the configuration manifold $Q, g(q)=\left[g_{i j}(q)\right]$ is bilinear, symmetric and positive definite, and $V(q)$ is the potential energy function on $Q$. The kinetic energy induces a Riemannian metric denoted by $\langle\langle\cdot, \cdot\rangle\rangle$ defined as $\left\langle\langle X, Y\rangle=\sum_{i, j=1}^{n} g_{i j}(q) X^{i} Y^{j}\right.$, where $X$ and $Y$ are two vector fields on $Q$. Hence, the Lagrangian can be written as $L(q, \dot{q})=$ $\frac{1}{2}\langle\dot{q}, \dot{q}\rangle-V(q)$, where $\dot{q}=\left(\dot{q}_{1}, \ldots, \dot{q}_{n}\right)$.

The equations of motion in local coordinates are given by

$$
\frac{\mathrm{d}}{\mathrm{d} t}\left(\frac{\partial L}{\partial \dot{q}_{i}}\right)-\frac{\partial L}{\partial q_{i}}=b_{i j}(q) u^{j}, \quad i=1, \ldots, n
$$

where $u^{i}, i=1, \ldots, m$, are control inputs. Letting $v^{i}=\dot{q}^{i}$, the equations of motion can be expressed as

$$
\begin{aligned}
& \dot{q}^{i}=v^{i} \\
& \dot{v}^{i}=-\Gamma_{j k}^{i}(q) v^{j} v^{k}+\operatorname{grad} V(q)+\sum_{j=1}^{m} Y_{j}^{i}(q) u^{j}, \quad i=1, \ldots, n
\end{aligned}
$$

where the $i$ th element of the potential vector field $\operatorname{grad} V$ is $\operatorname{grad} V^{i}(q)=-g^{i j}(q)\left(\partial V(q) / \partial q_{j}\right)$, the $i$ th element of the control vector field $Y_{j}$ is $Y_{j}^{i}(q)=g^{i k}(q) b_{k j}(q)$, and $\Gamma_{j k}^{i}(q)$ are the Christoffel symbols computed from the metric $g(q)$ and satisfy $\Gamma_{j k}^{i}=\Gamma_{k j}^{i}$, due to the symmetry of $g(q)$. For a vector field $X(q)=X^{i}(q)\left(\partial / \partial q^{i}\right)$ on $Q$, we define its vertical lift on $T Q$ as $X^{\text {lift }}\left(v_{q}\right)=X^{i}(q)\left(\partial / \partial v^{i}\right)$. Moreover, let $Z_{g}=v^{i}\left(\partial / \partial q^{i}\right)-\Gamma_{j k}^{i} v^{j} v^{k}\left(\partial / \partial v^{i}\right)$ in the subsequent development.

The symmetric product of two vector fields $X$ and $Y$ on $Q$ is defined as $\langle X: Y\rangle=\nabla_{X} Y+\nabla_{Y} X$, where $\nabla_{X} Y$ represents the covariant derivative of $Y$ with respect to $X$. The local coordinate expression for $\langle X: Y\rangle$ is

$$
\langle X: Y\rangle=\left(\frac{\partial X^{i}}{\partial q_{j}} Y^{j}+\frac{\partial Y^{i}}{\partial q_{j}} X^{j}+\Gamma_{j k}^{i} X^{j} Y^{k}+\Gamma_{k j}^{i} X^{k} Y^{j}\right) \frac{\partial}{\partial q_{i}}
$$


It is shown in [13] that when iterated Lie brackets generated from $\left\{Z_{g}+\operatorname{grad} V^{\text {lift }}, Y_{1}^{\text {lift }}, \ldots, Y_{m}^{\text {lift }}\right\}$ are evaluated on the zero section of $T Q$, quite a large number of the Lie brackets are zero. Those nontrivial Lie brackets are determined by the combinations of iterated symmetric products and Lie brackets generated from $\{\mathscr{Y} \cup \operatorname{grad} V\}$ as well as their lifts, where $\mathscr{Y}=\left\{Y_{1}, \ldots, Y_{m}\right\}$ is a family of control vector fields on $Q$. Therefore, controllability conditions can be expressed in terms of these symmetric products and Lie brackets. We call an iterated symmetric product from $\{\mathscr{Y} \cup \operatorname{grad} V\}$ bad if it contains an even number of each of the control vector fields $Y_{i}, i=1, \ldots, m$. Otherwise, we call it good. See [18] for the key early work in this regard. The degree of a symmetric product from $\{\mathscr{Y} \cup \operatorname{grad} V\}$ is the sum of the number of vector fields appearing in that symmetric product.

\subsection{Local configuration controllability and local equilibrium controllability}

For mechanical systems, it makes sense to consider the set of configurations that are reachable as opposed to the reachable set within the complete phase space. Following [13], we take local configuration accessibility to mean that starting from rest, one can reach an open set of configurations. By small-time local configuration controllability (STLCC), we mean that starting from rest at a given configuration, it is possible to reach a neighborhood of this given configuration (in small time). Finally, by local equilibrium controllability [19], we mean that from an equilibrium, it is possible to arrive at any other equilibrium in a small neighborhood of the original equilibrium; see the formal definition below. Now let $C_{\text {hor }}(\mathscr{Y}, V)$ be the projection of the distribution generated from $\{\mathscr{Y} \cup \operatorname{grad} V\}$ onto $T Q$; see [13] for an algorithm for computing $C_{\text {hor }}(\mathscr{Y}, V)$. Sufficient conditions for local configuration accessibility at $q$ and STLCC at $q$ for a mechanical system with the zero initial velocity are given below.

Theorem 1 (Lewis and Murray [13])

The mechanical control system (1)-(2) is locally configuration accessible at $q$ if $\operatorname{rank}\left(C_{\text {hor }}(\mathscr{Y}\right.$, $V)(q))=\operatorname{dim} Q$. Moreover, if the system is locally configuration accessible at $q$ and every bad symmetric product from $\{\mathscr{Y} \cup \operatorname{grad} V\}$ is a linear combination of lower degree good symmetric products, then the system is STLCC at $q$.

A useful extension of configuration controllability via Theorem 1 is (local) equilibrium controllability [13, 19]. Generally speaking, a mechanical system (1)-(2) is locally equilibrium controllable at an equilibrium $q_{e} \in Q$ if for each sufficiently small neighborhood of $q_{e}$, and for every equilibrium $\bar{q}_{e}$ in such a neighborhood, there exist $T>0$ and a solution-control pair $(c, u)$, where $c:[0, T] \rightarrow Q$, such that $c(0)=q_{e}, c(T)=\bar{q}_{e}$ with $\dot{c}(0)=0, \dot{c}(T)=0$. Note that if there is no potential term, i.e. grad $V \equiv 0$, any configuration is an equilibrium (for zero control inputs), but if the potential term exists, equilibrium points may be isolated. It is pointed out in [13] that the sufficient conditions in Theorem 1 imply stronger controllability results.

Corollary 2

If $q_{e} \in Q$ is an equilibrium and the hypotheses of Theorem 1 hold, then the mechanical control system (1)-(2) is locally equilibrium controllable at $q_{e}$.

Another useful extension is local fiber equilibrium controllability [17, 19] for a mechanical control system with a trivial principal fiber bundle structure [11], i.e. the configuration space $Q$ can be decomposed into $G \times Q_{s}$, where $G$ is the fiber space and $Q_{s}$ is the base space. Let $\tau$ : $Q \rightarrow G$ be the natural projection onto the fiber. Roughly speaking, an equilibrium $q_{e}=\left(g_{e}, r_{e}\right) \in Q$ is locally fiber equilibrium controllable, if for any small neighborhood of $q_{e}$ and any equilibrium 
$\bar{q}_{e}=\left(\bar{g}_{e}, \bar{r}_{e}\right)$ in such a neighborhood, a scalar $T>0$ and a solution-control pair $(c, u)$ exist, where $c:[0, T] \rightarrow Q$, such that $c(0)=q_{e}, \tau(c(T))=\bar{g}_{e}$ with $\dot{c}(0)=0, \dot{c}(T)=0$.

This general framework for local configuration controllability and local equilibrium controllability is extended to mechanical systems with nonholonomic constraints in [14]. A key observation is that in constrained systems, symmetric products can be defined and controllability can be checked in a similar manner as in unconstrained systems [14], but their formulation requires restricting and projecting the covariant derivative into the constraint distribution. Computing this covariant derivative is typically quite complicated, although some simplifications are made in [20] for certain class of mechanical systems. The Euler-Poincaré framework we consider in this paper has additional structure to explore so that we need not explicitly calculate this covariant derivative in developing controllability conditions.

\section{DYNAMICS OF CONSTRAINED MULTIBODY SYSTEMS}

In this section, a constrained multibody spherical robotic system is described. This system belongs to a general class of nonholonomic, multibody systems whose equations of motion are EulerPoincare given in the next subsection.

\subsection{Equations of motion and the equilibrium manifold of intermediate systems}

We start from the 'intermediate systems' [6], a class of multibody systems whose constraints take certain specific form that models rolling constraints in several physical examples. The spherical robot introduced soon falls into this class. Let $G$ be a matrix Lie group and $W$ be a vector space, and let $S$ denote the semidirect product $G(S) W$. The shape space $Q_{s}$ is an $n$-dimensional Abelian Lie group with local coordinates $r=\left(r_{1}, \ldots, r_{n}\right)$. Throughout the paper, we assume that controls act on the shape space $Q_{s}$ so that the shape is fully actuated. Physically speaking, the mechanical systems are assumed to be controlled via 'internal' actuation and shape change only. We also assume that $Q$ is a trivial principal fiber bundle, decomposed into the fiber space $S$ and the base space $Q_{s}$ that is fully controlled, and that for any $q=(g, r) \in G \times Q_{s}$, the left action of $G$ on $G \times Q_{s}$ is a smooth map $\Phi: G \times\left(G \times Q_{s}\right) \rightarrow\left(G \times Q_{s}\right)$ given by $\Phi_{h} q=(h g, r)$ for an arbitrary $h \in G$. Here, $\Phi$ is assumed to be free and proper. Consider a distribution $\mathscr{D}$ that is constructed from a smooth vector-valued function $\zeta: W \rightarrow W$, a fixed vector $a_{0} \in W$ and the action of $\mathfrak{g}$ on $W$ :

$$
\mathscr{D}_{(g, y)}=\left\{(\dot{g}, \dot{y}, \dot{r}) \in T Q: \dot{y}=\xi g \zeta\left(g^{-1} a_{0}\right)\right\}
$$

where $\xi=g^{-1} \dot{g} \in \mathfrak{g}$, and $y(t)$ is a (smooth) curve in $W$. We call $(Q, L, \mathscr{D})$ an intermediate (multibody) system [6], where $L$ is the Lagrangian on $Q$. Let $\Gamma=g^{-1} a_{0} \in W$ for a fixed $a_{0} \in W$, and $Y=g^{-1} \dot{y} \in W$. Following [6], we obtain the reduced Lagrangian as

$$
l(\xi, Y, \Gamma, r, \dot{r})=T(\xi, Y, r, \dot{r})-V(\Gamma, r)
$$

Suppose the reduced kinetic energy $T(\xi, Y, r, \dot{r})$ can be written as

$$
T(\xi, Y, r, \dot{r})=\frac{1}{2}\left[\begin{array}{lll}
\xi^{\mathrm{T}} & Y^{\mathrm{T}} & \dot{r}^{\mathrm{T}}
\end{array}\right] \bar{M}(r)\left[\begin{array}{c}
\xi \\
Y \\
\dot{r}
\end{array}\right]
$$


where $\bar{M}(r)$ denotes a reduced inertia tensor on $Q_{s}$ only, and the reduced constraint can be written as $Y=\xi \zeta(\Gamma)$ identified as a vector, where $\zeta: W \rightarrow W$ is a smooth vector-valued function. Thus, the constrained reduced Lagrangian becomes

$$
l_{c}(\xi, \Gamma, r, \dot{r})=T_{c}(\xi, \Gamma, r, \dot{r})-V(\Gamma, r)
$$

where

$$
T_{c}(\xi, \Gamma, r, \dot{r})=\frac{1}{2}\left[\begin{array}{ll}
\xi^{\mathrm{T}} & \dot{r}^{\mathrm{T}}
\end{array}\right] M(\Gamma, r)\left[\begin{array}{c}
\xi \\
\dot{r}
\end{array}\right]
$$

Here, $M(\Gamma, r)$ defines another reduced inertia tensor dependent on $Q_{s}$ and the dynamic parameter $\Gamma \in W$, which is referred to as 'advected parameter' in the literature $[15,19]$. Since $\Gamma=g^{-1} a_{0}$ evolves on $G$, one may view $M(\Gamma, r)$ as a function on $G \times Q_{s}$.

For $\theta \in W^{*}$ and $a \in W$, let $\diamond: W^{*} \times W \rightarrow \mathfrak{g}^{*}$ be defined as $\langle\theta, \xi a\rangle=-\langle\theta \diamond a, \xi\rangle \forall \xi \in \mathfrak{g}$. In terms of $\diamond$, the Euler-Poincaré equation is

$$
\frac{\mathrm{d}}{\mathrm{d} t}\left(\frac{\partial l_{c}}{\partial \xi}\right)-\operatorname{ad}_{\xi}^{*}\left(\frac{\partial l_{c}}{\partial \xi}\right)=\frac{\partial l}{\partial \Gamma} \diamond \Gamma-\frac{\partial l}{\partial Y} \diamond\left(\frac{\mathrm{d}}{\mathrm{d} t} \zeta\right)
$$

where $Y$ in the argument of $\partial l / \partial \Gamma$ and $\partial l / \partial Y$ is evaluated along the constraints $Y=\xi \zeta(\Gamma)$, and $(\mathrm{d} / \mathrm{d} t) \zeta$ corresponds to $(\mathrm{d} / \mathrm{d} t) \zeta(\Gamma(t))$. The equations of motion for the shape dynamics are

$$
\frac{\mathrm{d}}{\mathrm{d} t}\left(\frac{\partial l_{c}}{\partial \dot{r}}\right)-\frac{\partial l_{c}}{\partial r}=u_{s}
$$

where $u_{s}$ denotes the shape control. Equations (3)-(4) and the advection equation $\dot{\Gamma}+\xi \Gamma=0$ form the reduced equations for the constrained systems of interest. These equations, together with the constraint equation $Y=g^{-1} \dot{y}=\xi \zeta(\Gamma)$, completely describe the system dynamics on $Q=S \times Q_{s}$.

Using the expressions for $l$ and $l_{c}$, we obtain the terms in (3) to be used in the subsequent development:

$$
\begin{gathered}
\left(\begin{array}{c}
\frac{\partial l_{c}}{\partial \xi} \\
\frac{\partial l_{c}}{\partial \dot{r}}
\end{array}\right)=M(\Gamma, r)\left[\begin{array}{c}
\xi \\
\dot{r}
\end{array}\right],\left.\quad \frac{\partial l}{\partial \Gamma}\right|_{Y=\xi \zeta(\Gamma)}=-\frac{\partial V(\Gamma, r)}{\partial \Gamma} \\
\left.\frac{\partial l}{\partial Y}\right|_{Y=\xi \zeta(\Gamma)}=H(\Gamma, r)\left[\begin{array}{c}
\xi \\
\dot{r}
\end{array}\right], \quad \frac{\mathrm{d}}{\mathrm{d} t} \zeta=\frac{\partial \zeta}{\partial \Gamma}(-\xi \Gamma)
\end{gathered}
$$

where $H(\Gamma, r): \mathfrak{g} \times T_{r} Q_{s} \rightarrow W^{*}$ is a smooth (matrix-valued) function on $G \times Q_{s}$, expressed in terms of components of $\bar{M}(r)$ and $\Gamma$.

Letting $\xi=0$ and $\dot{r}=0$, we obtain the conditions for a (controlled) equilibrium $q_{e}=\left(g_{e}, r_{e}\right)$ :

$$
\frac{\partial V\left(\Gamma_{e}, r_{e}\right)}{\partial \Gamma} \diamond \Gamma_{e}=0, \quad \frac{\partial V\left(\Gamma_{e}, r_{e}\right)}{\partial r}=u_{s e}
$$

where $\Gamma_{e}=g_{e}^{-1} a_{0}$, and $u_{s e}$ denotes a constant shape control input that maintains an arbitrary shape configuration $r_{e}$. Note that $u_{s e}$ is generally not zero. Moreover, $\left\{h \in G \mid \Gamma_{e}=h \Gamma_{e}\right\}$ is a 
symmetry (or isotropy) group of $\Gamma_{e}$. The symmetry (or isotropy) algebra at $\Gamma_{e}$ is $\left\{\xi \in \mathfrak{g} \mid \xi \Gamma_{e}=0\right\}$. It is easy to verify that $S_{e}=\left\{\left(g_{e} h^{-1}, r_{e}\right) \in G \times Q_{s} \mid \Gamma_{e}=h \Gamma_{e}\right\}$ is a set of (controlled) equilibrium configurations at $r_{e}$. We call $S_{e}$ the (controlled) equilibrium configuration set associated with $r_{e}$.

Let $q=(g, r) \in G \times Q_{s}$ and let $X$ be a vector field on $G \times Q_{s}$ of the form $X=\left(g \xi_{X}(q), v_{X}(q)\right)$. In the following, we use $\widetilde{X}$ or $X^{\sim}$ to denote the left translation of $X$ to the identity of $G$, that is, $\widetilde{X}(q)=X^{\sim}(q):=T_{q} \Phi_{g^{-1}}\left(g \xi_{X}(q), v_{X}(q)\right)=\left(\xi_{X}(q), v_{X}(q)\right)$, where both $\xi_{X}$ and $v_{X}$ are treated as vector-valued functions. For the reduced system (3)-(4) on $G \times Q_{s}$, the control vector fields are given by

$$
\tilde{Y}_{i}=M^{-1}(\Gamma, r)\left[\begin{array}{c}
0 \\
Y_{i s}
\end{array}\right], \quad i=1, \ldots, n
$$

where $Y_{i s}$ is a $T^{*} Q_{s}$-valued function such that $\left\{Y_{1 s}, \ldots, Y_{n s}\right\}$ span $T^{*} Q_{s}$, based on the full shape actuation assumption. Let $\mathscr{Y}=\left\{Y_{1}, \ldots, Y_{n}\right\}$ be a family of the control vector fields. Since the shape is fully actuated, a control transformation can be introduced such that the transformed potential vector field becomes

$$
(\operatorname{grad} V)^{\sim}=M^{-1}(\Gamma, r)\left[\begin{array}{c}
-\frac{\partial V(\Gamma, r)}{\partial \Gamma} \diamond \Gamma \\
0
\end{array}\right]
$$

which implies that $\operatorname{grad} V\left(q_{e}\right)=0$ at an equilibrium configuration $q_{e}$. This transformation does not affect symmetric product spanning relations, and thus does not change controllability results either.

\subsection{Equations of motion and equilibrium conditions of the spherical robot}

We apply the general results in the previous subsection to a spherical robot controlled by internal actuators such as rotors and sliders; see Figure 1 for its schematic configuration. The spherical base body of the robot can roll without sliding on a horizontal plane in a uniform gravitational field. Such a robot can be viewed as a controlled Chaplygin's sphere or Chaplygin's top.

Choose a base body coordinate frame with the origin at the center of the ball. Let $x \in \mathbb{R}^{3}$ denote the position of the center of the ball in the inertial frame, and let $R \in S O$ (3) represent the base body attitude that maps from the base body coordinate frame onto the inertial frame. Relative motion of the internal actuators with respect to the base body is described by generalized shape coordinates $r \in Q_{s}$, where $Q_{s}$ is referred to as the shape space. Hence, the configuration space manifold is $S O(3) \times \mathbb{R}^{3} \times Q_{s}$. Note that we are only interested in $\left(x_{1}, x_{2}\right)$, the horizontal position of the center of the ball.

It is noted that $G=S O(3), W=\mathbb{R}^{3}$, and $a_{0}=e_{3}$ for the spherical robot. Let $a$ be the radius of the spherical base body and $m_{0}$ be its mass, $J_{0}$ be the inertia tensor of the base body defined with respect to the base body coordinate frame, $m_{i}, i=1, \ldots, N$, be the mass of the $i$ th auxiliary body, and $J_{i}(r)$ be the inertia tensor of the $i$ th auxiliary body defined with respect to the base body coordinate frame. Moreover, let $\rho_{0}$ denote the relative position vector of the center of mass of the ball and let $\rho_{i}(r)$ denote the relative position vector of the center of mass of the $i$ th auxiliary body. We denote the angular velocity of the $i$ th body relative to the base body coordinate frame by $C_{i}(r) \dot{r}$. That is, suppose the orientation of the $i$ th body in the base body frame is given by $R_{i}(r) \in S O(3)$, where $R_{i}(r)$ maps from a coordinate frame for the $i$ th body onto the base body 




Figure 1. Schematic configuration of a spherical robot in a uniform gravitational field with a slider and a rotor.

coordinate frame. Let $\widehat{\omega}_{i}(r, \dot{r})=R_{i}^{-1}(r)(\mathrm{d} / \mathrm{d} t) R_{i}(r)$ denote the angular velocity of the $i$ th body relative to its own coordinate frame. Then $C_{i}(r) \dot{r}=R_{i}(r) \omega_{i}(r, \dot{r})$, see [15] for details.

Let $v=R^{-1} \dot{x}$ and $\widehat{\omega}=R^{-1} \dot{R}$ denote the linear and angular velocities of the base body expressed in the base body frame, respectively. The reduced kinetic energy is

$$
T(v, \omega, r, \dot{r})=\frac{1}{2}\left[\begin{array}{lll}
v^{\mathrm{T}} & \omega^{\mathrm{T}} & \dot{r}^{\mathrm{T}}
\end{array}\right] \bar{M}(r)\left[\begin{array}{c}
v \\
\omega \\
\dot{r}
\end{array}\right]
$$

where

$$
\bar{M}(r)=\left[\begin{array}{ccc}
m_{T} I_{3} & K(r) & B_{t}(r) \\
K^{\mathrm{T}}(r) & J(r) & B_{r}(r) \\
B_{t}^{\mathrm{T}}(r) & B_{r}^{\mathrm{T}}(r) & m(r)
\end{array}\right]
$$

is symmetric and positive definite for all $r \in Q_{s}, m_{T}=\sum_{i=0}^{N} m_{i}$ is the total mass, $K(r)=-\sum_{i=0}^{N} m_{i} \widehat{\rho}_{i}(r), J(r)=\sum_{i=0}^{N}\left\{J_{i}(r)-m_{i} \widehat{\rho}_{i}(r) \widehat{\rho}_{i}(r)\right\}, B_{t}(r)=\sum_{i=1}^{N} m_{i} \partial \rho_{i}(r) / \partial r$, and

$$
\begin{aligned}
& B_{r}(r)=\sum_{i=1}^{N}\left\{m_{i} \widehat{\rho}_{i}(r)\left[\frac{\partial \rho_{i}(r)}{\partial r}\right]+J_{i}(r) C_{i}(r)\right\} \\
& m(r)=\sum_{i=1}^{N}\left\{m_{i}\left[\frac{\partial \rho_{i}(r)}{\partial r}\right]^{\mathrm{T}}\left[\frac{\partial \rho_{i}(r)}{\partial r}\right]+C_{i}^{\mathrm{T}}(r) J_{i}(r) C_{i}(r)\right\}
\end{aligned}
$$

Let $\rho_{c}(r)$ denote the position vector of the center of mass of the multibody system with respect to the base body coordinate frame, which is given by $\rho_{c}(r)=1 / m_{T}\left(\sum_{i=0}^{N} m_{i} \rho_{i}(r)\right)$. Thus, $K(r)=-m_{T} \widehat{\rho}_{c}(r), B_{t}(r)=m_{T}\left(\partial \rho_{c}(r) / \partial r\right)$. 
Let $\Gamma=R^{\mathrm{T}} e_{3}$, which satisfies $\dot{\Gamma}=\Gamma \times \omega$. The reduced gravitational potential energy, measured from the origin of the body frame (i.e. the geometric center of the ball), can be expressed as $V(\Gamma, r)=m_{T} a_{g} \Gamma \cdot \rho_{c}(r)$, where $a_{g}$ is the gravity constant. The reduced constraint equation, followed from the assumption that the ball rolls without sliding, is given by $v=a \omega \times \Gamma$. Substituting the constraint equation into the reduced kinetic energy, we obtain

$$
T_{c}(\omega, \Gamma, r, \dot{r})=\frac{1}{2}\left[\begin{array}{ll}
\omega^{\mathrm{T}} & \dot{r}^{\mathrm{T}}
\end{array}\right] \underbrace{\left[\begin{array}{ll}
M_{11}(\Gamma, r) & M_{12}(\Gamma, r) \\
M_{21}(\Gamma, r) & M_{22}(r)
\end{array}\right]}_{M(\Gamma, r)}\left[\begin{array}{c}
\omega \\
\dot{r}
\end{array}\right]
$$

where $M_{11}(\Gamma, r)=\sum_{i=0}^{N}\left(J_{i}(r)-m_{i}\left[\rho_{i}(r)+a \Gamma\right]^{\wedge}\left[\rho_{i}(r)+a \Gamma\right]^{\wedge}\right), M_{12}(\Gamma, r)=M_{21}^{\mathrm{T}}(\Gamma, r)=B_{r}(r)+$ $a \widehat{\Gamma} B_{t}(r)$, and $M_{22}(r)=m(r)$. Moreover, we have $\zeta(\Gamma)=a \Gamma$, and

$$
\begin{gathered}
\left.\frac{\partial l}{\partial v}\right|_{v=a \omega \times \Gamma}=\underbrace{\left[K(r)-m_{T} a \widehat{\Gamma}, B_{t}(r)\right]}_{H(\Gamma, r)}\left[\begin{array}{c}
\omega \\
\dot{r}
\end{array}\right]=\underbrace{\left[-m_{T}\left[\rho_{c}(r)+a \Gamma\right]^{\wedge}, m_{T} \frac{\partial \rho_{c}(r)}{\partial r}\right.}_{H(\Gamma, r)}\left[\begin{array}{c}
\omega \\
\dot{r}
\end{array}\right] \\
\frac{\mathrm{d}}{\mathrm{d} t} \zeta=\frac{\mathrm{d}(a \Gamma)}{\mathrm{d} t}=a \Gamma \times \omega,\left.\quad \frac{\partial l}{\partial \Gamma}\right|_{v=a \omega \times \Gamma}=-m_{T} a_{g} \rho_{c}(r)
\end{gathered}
$$

It can further be verified that for $v \in W^{*}=\mathbb{R}^{3}$ and $u \in W=\mathbb{R}^{3}$, the operation $\diamond$ is identified with the cross-product, i.e. $v \diamond u=v \times u$. This yields, after suitable simplification,

$$
\begin{aligned}
\left.\frac{\partial l}{\partial v}\right|_{v=a \omega \times \Gamma} \diamond\left(\frac{d}{d t} \zeta\right) & =m_{T} a\left\{\left[\left(\frac{\partial \rho_{c}(r)}{\partial r} \dot{r}\right) \cdot \omega\right] \Gamma-\left[\left(\rho_{c}(r) \times \Gamma\right) \cdot \omega+\left(\frac{\partial \rho_{c}(r)}{\partial r} \dot{r}\right) \cdot \Gamma\right] \omega\right\} \\
\left.\frac{\partial l}{\partial \Gamma}\right|_{v=a \omega \times \Gamma} \diamond \Gamma & =m_{T} a_{g} \Gamma \times \rho_{c}(r)
\end{aligned}
$$

Substituting the above results into (3) - (4), we obtain the reduced equations of motion on $S O(3) \times Q_{s}$ :

$$
\begin{aligned}
\frac{\mathrm{d}}{\mathrm{d} t}\left(\frac{\partial l_{c}}{\partial \omega}\right)-\frac{\partial l_{c}}{\partial \omega} \times \omega= & -m_{T} a\left\{\left[\left(\frac{\partial \rho_{c}(r)}{\partial r} \dot{r}\right) \cdot \omega\right] \Gamma-\left[\left(\rho_{c}(r) \times \Gamma\right) \cdot \omega\right.\right. \\
& \left.\left.+\left(\frac{\partial \rho_{c}(r)}{\partial r} \dot{r}\right) \cdot \Gamma\right] \omega\right\}+m_{T} a_{g} \Gamma \times \rho_{c}(r) \\
\frac{\mathrm{d}}{\mathrm{d} t}\left(\frac{\partial l_{c}}{\partial \dot{r}}\right)-\frac{\partial l_{c}}{\partial r}= & u_{S}
\end{aligned}
$$

where the shape dynamics are fully controlled. It is clear that the controlled equilibrium of the reduced system is given by $\left\{(r, R) \mid \Gamma \times \rho_{c}(r)=0\right\}$, where $\Gamma=R^{\mathrm{T}} e_{3}$. Furthermore, we consider two types of control vector fields subsequently

$$
\tilde{Y}_{i}=M^{-1}(\Gamma, r)\left[\begin{array}{l}
0 \\
e_{i}
\end{array}\right] \text { or } \tilde{Y}_{i}^{A}=\left[\begin{array}{c}
-A_{i}(\Gamma, r) \\
e_{i}
\end{array}\right]=M^{-1}(\Gamma, r)\left[\begin{array}{c}
0 \\
\Delta(\Gamma, r) e_{i}
\end{array}\right], i=1, \ldots, n
$$


where $\Delta=M_{22}-M_{21} M_{11}^{-1} M_{12}$ and $A_{i}$ is the $i$ th column of $A(\Gamma, r)=M_{11}^{-1}(\Gamma, r) M_{12}(\Gamma, r)$. The control vector fields $\widetilde{Y}_{i}^{A}$ correspond to those via a control transformation discussed before (8). And the transformed gravitational potential vector field in this case is given by

$$
(\operatorname{grad} V)^{\sim}=m_{T} a_{g} M^{-1}(\Gamma, r)\left[\begin{array}{c}
\Gamma \times \rho_{c}(r) \\
0
\end{array}\right]
$$

\section{CONTROLLABILITY OF CONSTRAINED MULTIBODY SYSTEMS}

In this section, we exploit Lie bracket and symmetric product tools developed in the above section for controllability analysis of the constrained multibody systems that fit into the class of intermediate systems introduced in Section 3.1.

\subsection{Lie brackets and symmetric products on $G \times Q_{s}$}

We focus on the reduced dynamics on $G \times Q_{s}$ described by (3)-(4) and derive essential results on Lie brackets and symmetric products on $G \times Q_{s}$ first. These results will be used for controllability analysis on the full configuration space $Q=S \times Q_{s}=(G \subseteq W) \times Q_{s}$ in Section 4.2. Let $q=(g, r) \in G \times Q_{s}$ and let $X=\left(g \xi_{X}, v_{X}\right)$ and $Z=\left(g \xi_{Z}, v_{Z}\right)$ be two vector fields on $G \times Q_{s}$. The Lie bracket of $X$ and $Z$ is given by [19]

$$
[X, Z]^{\sim}(q)=\left(\operatorname{ad}_{X} Z\right)^{\sim}(q)+\left.\frac{\mathrm{d}}{\mathrm{d} t}\right|_{t=0}\left(\widetilde{Z} \circ \phi_{t}^{X}(q)-\tilde{X} \circ \phi_{t}^{Z}(q)\right)
$$

where $\phi_{t}^{X}(q)$ and $\phi_{t}^{Z}(q)$ denote the flows of the vector fields $X$ and $Z$ on $G \times Q_{s}$ starting from $q=(g, r)$, respectively, and

$$
\left(\operatorname{ad}_{X} Z\right)^{\sim}(q)=\left[\begin{array}{c}
\operatorname{ad}_{\xi_{X}} \xi_{Z}(q) \\
0
\end{array}\right]
$$

denotes the adjoint operation, and $\operatorname{ad}_{\xi_{X}} \xi_{Z}(q)$ is the adjoint operator on the Lie algebra $\mathfrak{g}$. Note that one may express the Lie bracket (14) in terms of coordinate-free tensor notation instead of using the flow notation $\phi_{t}^{X}$ and $\phi_{t}^{Z}$. However, the flow notation is adopted here for a more concise expression.

We apply (3)-(4) to derive the symmetric product formula. It should be noted that if the second term on the right-hand side of the Euler-Poincaré equation (3) vanishes, i.e. $\partial l / \partial Y \diamond((\mathrm{d} / \mathrm{d} t) \zeta) \equiv 0$, then the symmetric product is exactly the same as that derived in [19] except that the reduced inertia tensor is replaced by $M(\Gamma, r)$. However, because of these extra terms, it can be verified that the new affine connection that restricts to the constrained distribution may not be torsion free. We now compute the extra terms in the symmetric product of the vector fields $X$ and $Z$ due to the presence of $\partial l / \partial Y \diamond((\mathrm{d} / \mathrm{d} t) \zeta)$. According to (5)-(6), it is easy to verify that the extra terms are given by

$$
M^{-1}(\Gamma, r) \tilde{\Xi}(\tilde{X}, \widetilde{Z}):=M^{-1}(\Gamma, r)\left(\begin{array}{c}
\tilde{\rho}^{*}(\tilde{X}, \widetilde{Z})+\tilde{\rho}^{*}(\widetilde{Z}, \widetilde{X}) \\
0
\end{array}\right)
$$


where $\tilde{\rho}^{*}(\tilde{X}, \widetilde{Z})=H(\Gamma, r) \widetilde{Z} \diamond \partial \zeta / \partial \Gamma\left(-\xi_{X} \Gamma\right)$. Consequently, adding this extra term to the symmetric product formula derived in [19], we obtain the following symmetric product formula for the vector fields $X$ and $Z$ :

$$
\begin{aligned}
\langle X: Z\rangle^{\sim}(q)= & \left.\frac{\mathrm{d}}{\mathrm{d} t}\right|_{t=0}\left(\tilde{X} \circ \phi_{t}^{Z}(q)+\widetilde{Z} \circ \phi_{t}^{X}(q)\right)+M^{-1}(\Gamma, r)\left\{\left(\left.\frac{\mathrm{d}}{\mathrm{d} t}\right|_{t=0} M \circ \phi_{t}^{Z}(q)\right) \tilde{X}(q)\right. \\
& +\left(\left.\frac{\mathrm{d}}{\mathrm{d} t}\right|_{t=0} M \circ \phi_{t}^{X}(q)\right) \widetilde{Z}(q)-D M(\widetilde{X}(q), \widetilde{Z}(q))-\operatorname{ad}_{\widetilde{X}(q)}^{*} M(\Gamma, r) \widetilde{Z}(q) \\
& \left.-\operatorname{ad}_{\widetilde{Z}(q)}^{*} M(\Gamma, r) \widetilde{X}(q)+\tilde{\Xi}(\tilde{X}, \widetilde{Z})\right\}
\end{aligned}
$$

where $D M(\tilde{X}(q), \widetilde{Z}(q))=(\underbrace{0, \ldots, 0}_{\operatorname{dim}(G)}, D M_{s}(\tilde{X}(q), \widetilde{Z}(q))^{\mathrm{T}})^{\mathrm{T}}$, and

$$
D M_{s}(\widetilde{X}(q), \widetilde{Z}(q))=\left(\tilde{X}^{\mathrm{T}}(q) \frac{\partial M(\Gamma, r)}{\partial r_{1}} \widetilde{Z}(q), \ldots, \widetilde{X}^{\mathrm{T}}(q) \frac{\partial M(\Gamma, r)}{\partial r_{n}} \widetilde{Z}(q)\right)^{\mathrm{T}}
$$

This formula can also be obtained via a tedious computation using the definition of a symmetric product.

Consider two vector fields $X(q)$ and $Z(q)$ such that

$$
\tilde{X}(q)=\left[\begin{array}{c}
\xi_{X}(q) \\
v_{X}(q)
\end{array}\right]=M^{-1}(\Gamma, r)\left[\begin{array}{l}
X_{g}(q) \\
X_{s}(q)
\end{array}\right], \quad \widetilde{Z}(q)=\left[\begin{array}{c}
\xi_{Z}(q) \\
v_{Z}(q)
\end{array}\right]=M^{-1}(\Gamma, r)\left[\begin{array}{c}
Z_{g}(q) \\
Z_{s}(q)
\end{array}\right]
$$

where $X_{g}$ and $Z_{g}$ are $\mathfrak{g}^{*}$-valued functions, and $X_{S}$ and $Z_{s}$ are $T^{*} Q_{s}$-valued functions, all on $G \times Q_{s}$. Following [19], we obtain

$$
\langle X: Z\rangle^{\sim}(q)=M^{-1}(\Gamma, r)\left[\begin{array}{l}
\langle X: Z\rangle_{g}(q) \\
\langle X: Z\rangle_{s}(q)
\end{array}\right]
$$

where

$$
\begin{aligned}
\langle X: Z\rangle_{g}(q)= & \left.\frac{\mathrm{d}}{\mathrm{d} t}\right|_{t=0}\left(X_{g} \circ \phi_{t}^{Z}(q)+Z_{g} \circ \phi_{t}^{X}(q)\right)-\operatorname{ad}_{\xi_{X}(q)}^{*} Z_{g}(q)-\operatorname{ad}_{\xi_{Z}(q)}^{*} X_{g}(q) \\
& +\tilde{\rho}^{*}(\tilde{X}, \widetilde{Z})+\tilde{\rho}^{*}(\widetilde{Z}, \tilde{X}) \\
\langle X: Z\rangle_{s}(q)= & \left.\frac{\mathrm{d}}{\mathrm{d} t}\right|_{t=0}\left(X_{s} \circ \phi_{t}^{Z}(q)+Z_{s} \circ \phi_{t}^{X}(q)\right)-D M_{s}(\tilde{X}(q), \widetilde{Z}(q))
\end{aligned}
$$

Using the symmetric product formula (16), we have the following properties of symmetric products generated from $\{\mathscr{Y} \cup \operatorname{grad} V\}$ for the reduced dynamics (3)-(4), where the control vector fields $Y_{i} \in \mathscr{Y}$ and the potential vector field grad $V$ are given in (7) and (8), respectively.

1. Let $H=\{h \in G \mid \Gamma=h \Gamma\}$ be symmetry group of $\Gamma$, which is a subgroup of $G$. Thus, all the iterated symmetric products are invariant under $H$. 
2. Iterated symmetric products involving grad $V$ only are zero when evaluated at an equilibrium where $\operatorname{grad} V$ is evaluated as zero.

\section{Remark 3}

It is interesting to compare the above symmetric products with those dependent on the advected parameter $\Gamma$ for an unconstrained system studied in [19], under the same shape actuation assumptions. In that case, the shape control vector fields and the potential vector fields have a similar form to those in the constrained case. However, the reduced inertia tensor $M$ is on $Q_{s}$ only, and the control vector fields $Y_{i}$ are $G$-invariant and are in horizontal space determined by the reduced metric. It is shown in [19] that iterated symmetric products only involving the shape control vector fields are still $G$-invariant and horizontal. Moreover, an iterated symmetric product $Z$ involving $\operatorname{grad} V$ has the form

$$
\widetilde{Z}=M^{-1}(r)\left[\begin{array}{c}
G_{Z}(\Gamma, r) \diamond \Gamma \\
Z_{s}(\Gamma, r)
\end{array}\right]
$$

where $G_{Z}(\Gamma, r)$ is a $\mathfrak{g}^{*}$-valued function. It should be noted that these properties do not hold in the constrained case here because of dependence of the reduced inertia tensor $M$ on $\Gamma$ and the extra terms (15) in the symmetric product formula, which are due to the constraint.

\subsection{Controllability of the complete configuration $S \times Q_{s}$}

This section applies the Lie bracket and symmetric product results to local configuration/ equilibrium controllability analysis of the complete configuration space $S \times Q_{s}=(G \subseteq W) \times Q_{s}$.

We first look at a more general setting. Consider a simple mechanical system with constraint whose (local) configuration coordinates $q \in Q$ can be split into $q=\left(q_{1}, q_{2}\right) \in Q=Q_{1} \times Q_{2}$, where $q_{1} \in Q_{1}$ and $q_{2} \in Q_{2}$. Suppose the equations of motion can be written as

$$
\begin{aligned}
& \dot{q}_{1}^{i}=v_{1}^{i} \\
& \dot{v}_{1}^{i}=-\Gamma_{j k}^{i}\left(q_{1}\right) v_{1}^{j} v_{1}^{k}+\operatorname{grad} V^{i}\left(q_{1}\right)+\sum_{j=1}^{m} Y_{j}^{i}\left(q_{1}\right) u^{j} \\
& \dot{q}_{2}^{i}=h_{j}^{i}\left(q_{1}\right) v_{1}^{j}
\end{aligned}
$$

where $\Gamma_{j k}^{i}\left(q_{1}\right)$ are the Christoffel symbols (for the kinetic energy metric on $T Q_{1}$ ) dependent only on $q_{1}$, and $h$ is a smooth function on $Q_{1}$. The first two equations (21a)-(21b) characterize a subsystem on $T Q_{1}$ which is also a simple mechanical system, while the last equation (21c) describes a kinematic constraint that is independent of $q_{2}$. Many systems with symmetry and constraints can be put in this form by choosing appropriate local coordinates. See more examples in [14].

Now we look at local configuration/equilibrium controllability. Recall that the vertical lift of a vector field $X(q)=X^{i}\left(q_{1}\right) \partial / \partial q_{1}^{i}$ on $Q_{1}$ is $X^{\mathrm{lift}}\left(v_{1}\right)=X^{i}\left(q_{1}\right) \partial / \partial v_{1}^{i}$. Furthermore, let

$$
Z_{g}=v_{1}^{i} \frac{\partial}{\partial q_{1}^{i}}-\Gamma_{j k}^{i}\left(q_{1}\right) v_{1}^{j} v_{1}^{k} \frac{\partial}{\partial v_{1}^{i}}+h_{j}^{i}\left(q_{1}\right) v_{1}^{j} \frac{\partial}{\partial q_{2}^{i}}
$$

The following lemma is easy to show. 


\section{Lemma 4}

Let two vector fields on $Q$ be

$$
X_{\alpha}=U_{\alpha}^{i}\left(q_{1}\right) \frac{\partial}{\partial q_{1}^{i}}+h_{j}^{i}\left(q_{1}\right) U_{\alpha}^{j}\left(q_{1}\right) \frac{\partial}{\partial q_{2}^{i}}, \quad \alpha=1,2
$$

and their vertical lifts on $T Q$ be

$$
X_{\alpha}^{\mathrm{lift}}=U_{\alpha}^{i}\left(q_{1}\right) \partial / \partial v_{1}^{i}+h_{j}^{i}\left(q_{1}\right) U_{\alpha}^{j}\left(q_{1}\right) \partial / \partial v_{2}^{i}
$$

Then $\left[X_{2}^{\text {lift }},\left[\bar{Z}_{g}, X_{1}^{\text {lift }}\right]\right]=\left\langle X_{1}: X_{2}\right\rangle_{Q}^{\text {lift }}$, where

$$
\left\langle X_{1}: X_{2}\right\rangle_{Q}=\left\langle U_{1}: U_{2}\right\rangle_{Q_{1}}^{i}\left(q_{1}\right) \frac{\partial}{\partial q_{1}^{i}}+h_{j}^{i}\left(q_{1}\right)\left\langle U_{1}: U_{2}\right\rangle_{Q_{1}}^{j}\left(q_{1}\right) \frac{\partial}{\partial q_{2}^{i}}
$$

Note that in (21a), $T_{0_{q}} T Q=T_{q} Q \oplus V_{0_{q}} T Q$ for each $q \in Q$, where $V_{0_{q}} T Q$ is a subspace of $T_{0_{q}} T Q$ tangent to the fiber of $T Q$ at $q$. Hence, Lemma 4, together with Lemma 5.8 and Proposition 5.9 in [13], yields

$$
D_{\overline{\operatorname{Lie}}\left\{\left(\bar{Z}_{g}+\overline{\operatorname{grad} V}^{\text {lift }}\right) \cup \bar{Y}^{\mathrm{lift}}\right\}}\left(0_{q}\right) \cap V_{0_{q}} T Q=\left(C_{\mathrm{ver}}(\overline{\mathscr{Y}}, \bar{V})(q)\right)^{\mathrm{lift}}
$$

and

$$
\left.\left.D_{\overline{\operatorname{Lie}}\left\{\left(\bar{Z}_{g}+\overline{\operatorname{grad} V}\right.\right.}^{\text {lift }}\right) \cup \bar{Y}^{\text {lift }}\right\}\left(0_{q}\right) \cap T_{q} Q=C_{\text {hor }}(\overline{\mathscr{Y}}, \bar{V})(q)
$$

where the distributions $C_{\text {ver }}(\overline{\mathscr{Y}}, \bar{V})(q)$ and $C_{\text {hor }}(\overline{\mathscr{Y}}, \bar{V})(q)$ satisfy the constraints and can be computed using Algorithm 7.1 in [13]. Moreover, Lemma 4 implies that the good-bad symmetric product condition of the complete system (21a)-(21c) holds if and only if the good-bad symmetric product condition of sub-system (21a)-(21b) holds. Hence, one only needs to compute the distribution $C_{\text {ver }}(\mathscr{Y}, V)$ of sub-system (21a)-(21b) on $Q_{1}$, which simplifies symmetric product computations. We summarize this analysis as follows.

\section{Proposition 5}

The following statements hold for system (21a).

1. If $C_{\text {hor }}(\bar{Y}, \bar{V})(q)=T_{q} Q$, then the system is locally configuration accessible at $q$.

2. If the system is locally configuration accessible and its subsystem (21a)-(21b) on $Q_{1}$ satisfies the good-bad symmetric product condition, then the system is locally configuration/ equilibrium controllable at $q$.

By noting that $\overline{\operatorname{Lie}}(\overline{\mathscr{Y}}) \subseteq \overline{\operatorname{Lie}}(\overline{\operatorname{Sym}}(\overline{\mathscr{Y}})) \subseteq C_{\text {hor }}(\overline{\mathscr{Y}}, \bar{V})$ [19], we obtain an easily verified (but conservative) sufficient condition for local configuration accessibility.

\section{Corollary 6}

If $\overline{\operatorname{Lie}}(\overline{\mathscr{Y}})(q)=T_{q} Q$, then the system is locally configuration accessible at $q$.

This corollary is much less conservative if $\operatorname{grad} V \equiv 0$. In this case, $C_{\text {hor }}(\overline{\mathscr{Y}}, \bar{V})=\overline{\operatorname{Lie}}(\overline{\operatorname{Sym}}(\overline{\mathscr{Y}}))$ [14]. Furthermore, suppose $\overline{\mathscr{Y}}$ and $\overline{\operatorname{Sym}}(\overline{\mathscr{Y}})$ are horizontal (defined via the Riemannian metric). Since the shape is fully actuated, we have $\overline{\operatorname{Sym}}(\overline{\mathscr{Y}})=\overline{\mathscr{Y}}[19]$, thus $\overline{\operatorname{Lie}}(\overline{\operatorname{Sym}}(\overline{\mathscr{Y}}))=\overline{\operatorname{Lie}}(\overline{\mathscr{Y}})$. Therefore, $C_{\text {hor }}(\overline{\mathscr{Y}}, \bar{V})=\overline{\operatorname{Lie}}(\overline{\mathscr{Y}})$ in such cases. 


\section{CONTROLLABILITY OF THE MULTIBODY SPHERICAL ROBOT}

The general results in the previous section are applied to the spherical robot controlled by internal rotors. Specific controllability conditions are derived for two different cases of this nontrivial example, i.e. a controlled Chaplygin's sphere and a controlled Chaplygin's top. We also discuss the Chaplygin's sphere and a Chaplygin's top controlled by sliders.

\subsection{Lie brackets and symmetric products}

Let $q=(R, r) \in S O(3) \times Q_{s}$ and $X(q), Z(q)$ be two vector fields on $S O(3) \times Q_{s}$ of the form

$$
\widetilde{X}(q)=\left[\begin{array}{c}
\omega_{X}(\Gamma, r) \\
v_{X}(\Gamma, r)
\end{array}\right], \quad \widetilde{Z}(q)=\left[\begin{array}{c}
\omega_{Z}(\Gamma, r) \\
v_{Z}(\Gamma, r)
\end{array}\right]
$$

where $\widehat{\omega}_{X}, \widehat{\omega}_{Z} \in \mathfrak{s v}(3)$. Such vector fields are invariant under the subgroup $H=\{R \in S O(3) \mid \Gamma=$ $R \Gamma\}$. The Lie bracket of $X(q)$ and $Z(q)$ is given by

$$
[X, Z]^{\sim}=\left[\begin{array}{c}
\omega_{X} \times \omega_{Z}+\frac{\partial \omega_{Z}}{\partial \Gamma}\left[\Gamma \times \omega_{X}\right]-\frac{\partial \omega_{X}}{\partial \Gamma}\left[\Gamma \times \omega_{Z}\right]+\frac{\partial \omega_{Z}}{\partial r} v_{X}-\frac{\partial \omega_{X}}{\partial r} v_{Z} \\
\frac{\partial v_{Z}}{\partial \Gamma}\left[\Gamma \times \omega_{X}\right]-\frac{\partial v_{X}}{\partial \Gamma}\left[\Gamma \times \omega_{Z}\right]+\frac{\partial v_{Z}}{\partial r} v_{X}-\frac{\partial v_{X}}{\partial r} v_{Z}
\end{array}\right]
$$

We now consider Lie brackets for vector fields on the complete configuration space $S O(3)$ $\times \mathbb{R}^{3} \times Q_{s}$. From the constraint equation $v=a \omega \times \Gamma$, we have $\dot{x}=-a e_{3} \times(R \omega)$. Thus, for two vector fields $\bar{X}$ and $\bar{Z}$ of the form

$$
\bar{X}=\left[\begin{array}{c}
R \widehat{\omega}_{X}(\Gamma, r) \\
v_{X}(\Gamma, r) \\
-a e_{3} \times\left[R \omega_{X}(\Gamma, r)\right]
\end{array}\right], \quad \bar{Z}=\left[\begin{array}{c}
R \widehat{\omega}_{Z}(\Gamma, r) \\
v_{Z}(\Gamma, r) \\
-a e_{3} \times\left[R \omega_{Z}(\Gamma, r)\right]
\end{array}\right]
$$

their Lie bracket is given by

$$
[\bar{X}, \bar{Z}]=\left[\begin{array}{c}
R\left[\omega_{X} \times \omega_{Z}+\frac{\partial \omega_{Z}}{\partial \Gamma}\left[\Gamma \times \omega_{X}\right]-\frac{\partial \omega_{X}}{\partial \Gamma}\left[\Gamma \times \omega_{Z}\right]+\frac{\partial \omega_{Z}}{\partial r} v_{X}-\frac{\partial \omega_{X}}{\partial r} v_{Z}\right]^{\wedge} \\
\frac{\partial v_{Z}}{\partial \Gamma}\left[\Gamma \times \omega_{X}\right]-\frac{\partial v_{X}}{\partial \Gamma}\left[\Gamma \times \omega_{Z}\right]+\frac{\partial v_{Z}}{\partial r} v_{X}-\frac{\partial v_{X}}{\partial r} v_{Z} \\
-a e_{3} \times R\left(2 \omega_{X} \times \omega_{Z}+\frac{\partial \omega_{Z}}{\partial \Gamma}\left[\Gamma \times \omega_{X}\right]-\frac{\partial \omega_{X}}{\partial \Gamma}\left[\Gamma \times \omega_{Z}\right]+\frac{\partial \omega_{Z}}{\partial r} v_{X}-\frac{\partial \omega_{X}}{\partial r} v_{Z}\right)
\end{array}\right]
$$

Note that the vertical component of the additional $\mathbb{R}^{3}$ factor is zero (i.e. $x_{3}=0$ ) for $\bar{X}, \bar{Z}$, and their Lie bracket, that is, the ball is constrained to roll on a horizontal plane. For notational and computational convenience, we identify vector fields of form (22) with a vector-valued function 
via a group translation in the following development. For example, $\bar{X}$ in (22) is identified as

$$
\bar{X} \simeq\left[\begin{array}{c}
\omega_{X}(\Gamma, r) \\
v_{X}(\Gamma, r) \\
\Gamma \times \omega_{X}(\Gamma, r)
\end{array}\right]
$$

This notation is consistent with that introduced at the beginning of Section 4.1 for the intermediate systems.

Finally, we briefly mention how to compute symmetric products. To avoid tedious computations, we focus only on the extra term due to the nonholonomic constraints. Using (15), it is easy to verify that for the two vector fields $X$ and $Z$ defined above, the extra term in the symmetric product is

$$
\begin{aligned}
\tilde{\rho}^{*}(\tilde{X}, \widetilde{Z})+\tilde{\rho}^{*}(\widetilde{Z}, \tilde{X})= & m_{T} a\left\{\left[\left(\frac{\partial \rho_{c}(r)}{\partial r} v_{Z}\right) \cdot \omega_{X}+\left(\frac{\partial \rho_{c}(r)}{\partial r} v_{X}\right) \cdot \omega_{Z}\right] \Gamma\right. \\
& -\left[\left(\rho_{c}(r) \times \Gamma\right) \cdot \omega_{Z}+\left(\frac{\partial \rho_{c}(r)}{\partial r} v_{Z}\right) \cdot \Gamma\right] \omega_{X} \\
& \left.-\left[\left(\rho_{c}(r) \times \Gamma\right) \cdot \omega_{X}+\left(\frac{\partial \rho_{c}(r)}{\partial r} v_{X}\right) \cdot \Gamma\right] \omega_{Z}\right\}
\end{aligned}
$$

Evaluated at an equilibrium where $\rho_{c}(r) \times \Gamma=0$, we have

$$
\begin{aligned}
\tilde{\rho}^{*}(\tilde{X}, \widetilde{Z})+\tilde{\rho}^{*}(\tilde{Z}, \tilde{X})= & m_{T} a\left\{\left[\left(\frac{\partial \rho_{c}(r)}{\partial r} v_{Z}\right) \cdot \omega_{X}+\left(\frac{\partial \rho_{c}(r)}{\partial r} v_{X}\right) \cdot \omega_{Z}\right] \Gamma\right. \\
& \left.-\left[\left(\frac{\partial \rho_{c}(r)}{\partial r} v_{Z}\right) \cdot \Gamma\right] \omega_{X}-\left[\left(\frac{\partial \rho_{c}(r)}{\partial r} v_{X}\right) \cdot \Gamma\right] \omega_{Z}\right\}
\end{aligned}
$$

which shall be used for symmetric product computation subsequently.

\subsection{Controllability of a spherical robot controlled by rotors}

Consider a spherical robot controlled by $n$ rotors, see Figure 2. The shape space in this case is $Q_{s}=\underbrace{\mathbb{S}^{1} \times \cdots \times \mathbb{S}^{1}}_{n}$. A feature of this system is that it is invariant with respect to the shape variable, e.g. $\bar{M}$ and $\rho_{c}$ are both constant and $B_{t}(r) \equiv 0$. Thus,

$$
M_{11}(\Gamma)=J-m_{T} a\left[a \widehat{\Gamma}^{2}+\widehat{\Gamma} \widehat{\rho}_{c}+\widehat{\rho}_{c} \widehat{\Gamma}\right], \quad M_{12}=B_{r}=\sum_{i=1}^{N} J_{i} C_{i}, \quad H(\Gamma)=-\left[m_{T}\left(a \Gamma+\rho_{c}\right)^{\wedge}, 0\right]
$$

where $J=\sum_{i=0}^{n}\left(J_{i}-m_{i} \widehat{\rho}_{i} \widehat{\rho}_{i}\right)$ is constant. This implies that $M$ depends on $\Gamma$ only, so does $A=M_{11}^{-1} M_{12}$. If the spinning directions of the rotors are linear independent, then the columns of $M_{12}$ are linearly independent; see [15] for more details. In such case, we call the motors 


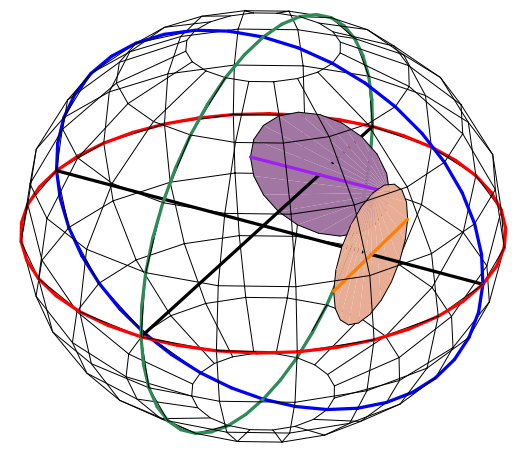

Figure 2. Schematic configuration of a spherical robot with two rotors.

'independent'. Consider the control vector fields $\tilde{Y}_{i}^{A}, i=1, \ldots, n$, of form (12). Following the Lie bracket formula in the previous section, we have

$$
\left[Y_{1}^{A}, Y_{2}^{A}\right]^{\sim}=\left[\begin{array}{c}
A_{1} \times A_{2}+\frac{\partial A_{2}}{\partial \Gamma}\left[\Gamma \times A_{1}\right]-\frac{\partial A_{1}}{\partial \Gamma}\left[\Gamma \times A_{2}\right] \\
0
\end{array}\right]
$$

Similarly, we can compute higher order Lie brackets. Note that the projection of all these Lie brackets onto $T_{r} Q_{s}$ is always zero because the vector fields are invariant under the translation on $Q_{s}$.

5.2.1. Chaplygin's sphere. We look at the case where the center of mass of the system is at the origin of the base body frame, i.e. the center of the ball. This implies that $\rho_{c} \equiv 0$ and the gravitational potential is identically zero. Hence,

$$
M_{11}(\Gamma)=J-m_{T} a^{2} \widehat{\Gamma}^{2}, \quad M_{12}=B_{r}=\sum_{i=1}^{N} J_{i} C_{i}, \quad H(\Gamma)=\left[-m_{T} a \widehat{\Gamma}, 0\right]
$$

The Euler-Poincaré equation and the shape equations on $S O(3) \times Q_{s}$ are simplified as

$$
\begin{aligned}
\frac{\mathrm{d}}{\mathrm{d} t}\left(\frac{\partial l_{c}}{\partial \omega}\right)-\frac{\partial l_{c}}{\partial \omega} \times \omega & =0 \\
\frac{\mathrm{d}}{\mathrm{d} t}\left(\frac{\partial l_{c}}{\partial \dot{r}}\right)-\frac{\partial l_{c}}{\partial r} & =u_{s}
\end{aligned}
$$

Obviously, all $(R, r) \in S O(3) \times Q_{s}$ are (controlled) equilibria. It should be noted that the dynamics in this case are similar to those without potential energy considered in [19], except that the reduced inertia tensor $M$ is dependent on $\Gamma$ (or more generally, on $R$ ).

There is an important conserved quantity in this case. Let $\Pi=\partial l_{c} / \partial \omega=M_{11}(\Gamma) \omega+M_{12} \dot{r}$ be the angular momentum conjugate to $\omega$. It is easy to verify using (24) that the inertial momentum $R \Pi$ is conserved. This is also shown in [6] for the one-body Chaplygin's sphere. Suppose the 
sphere is initially at equilibrium, and hence $R \Pi$ and thus $\Pi$ are identically zero; this leads to the relation $\omega=-A(\Gamma) \dot{r}$, where $A(\Gamma)=-M_{11}^{-1}(\Gamma) M_{12}$.

We now introduce more notation for the subsequent development. Let $\tau: S O(3) \times Q_{s} \rightarrow S O$ (3) be a projection, and let $\tau_{*}: T\left(S O(3) \times Q_{S}\right) \rightarrow T S O(3)$ denote its differential map. Based on the results in [19], we obtain the following results for local configuration accessibility of the reduced dynamics (24) $-(25)$ on $S O(3) \times Q_{s}$.

\section{Proposition 7}

Consider the reduced system (24)-(25) on $S O(3) \times Q_{s}$.

1. For the one-rotor case, i.e. $n=1$. The configuration accessibility condition fails and the system is not locally accessible.

2. For the two-rotor case, i.e. $n=2$. If $\tau_{*}\left\{\left[Y_{1}^{A}, Y_{2}^{A}\right],\left[Y_{1}^{A},\left[Y_{1}^{A}, Y_{2}^{A}\right]\right],\left[Y_{2}^{A},\left[Y_{1}^{A}, Y_{2}^{A}\right]\right]\right\}$ span $\mathfrak{s o}(3)$ at $R$, then system (24)-(25) is locally configuration accessible at $(R, r)$ for any $r \in Q_{s}$; if $\tau_{*}\left\{Y_{1}^{A}, Y_{2}^{A},\left[Y_{1}^{A}, Y_{2}^{A}\right]\right\}$ span $\mathfrak{s o}(3)$ at $R$, then system (24)-(25) is locally fiber configuration accessible at $(R, r)$ for any $r \in Q_{s}$.

3. For the three-rotor case, i.e. $n=3$. If $\tau_{*}\left\{\left[Y_{1}^{A}, Y_{2}^{A}\right],\left[Y_{1}^{A},\left[Y_{1}^{A}, Y_{2}^{A}\right]\right],\left[Y_{1}^{A}, Y_{3}^{A}\right]\right\}$ span sol(3) at $R$, then system (24)-(25) is locally configuration accessible at $(R, r)$ for any $r \in Q_{s}$; if $\tau_{*}\left\{Y_{1}^{A}, Y_{2}^{A}, Y_{3}^{A}\right\}$ span $\mathfrak{s p}(3)$ at $R$, then Equations (24)-(25) are locally fiber configuration accessible at $(R, r)$ for any $r \in Q_{s}$.

Since $\rho_{c} \equiv 0$, the extra term $\tilde{\rho}^{*}(\widetilde{X}, \widetilde{Z})+\tilde{\rho}^{*}(\widetilde{Z}, \widetilde{X})$ in symmetric product vanishes. Moreover, we only need to study symmetric products generated from the family of control vector fields $\mathscr{Y}$ due to $\operatorname{grad} V \equiv 0$. Following the symmetric product properties discussed in [19], we see that an iterated symmetric product $Z$ is always horizontal, i.e. it has the form

$$
\widetilde{Z}=M^{-1}(\Gamma)\left[\begin{array}{l}
0 \\
\star
\end{array}\right]
$$

Thus, the assumption that the shape dynamics are fully controlled implies that the good-bad symmetric product condition trivially holds on $S O(3) \times Q_{s}$.

We now look at controllability for the complete system on $Q=\left(S O(3) \mathbb{S} \mathbb{R}^{3}\right) \times Q_{s}$. Define

$$
\bar{Y}_{i}^{A}=\left[\begin{array}{c}
-R \widehat{A}_{i}(\Gamma) \\
e_{i} \\
a e_{3} \times\left[R A_{i}(\Gamma)\right]
\end{array}\right], \quad i=1, \ldots, n
$$

Iterated Lie brackets of $\left\{\bar{Y}_{1}^{A}, \ldots, \bar{Y}_{n}^{A}\right\}$ can be computed using (23). Local configuration accessibility results on $Q$ are as follows.

\section{Proposition 8}

Consider Chaplygin's sphere controlled by rotors.

1. Consider the sphere controlled by one rotor. Then the system is neither locally fiber configuration accessible nor small-time locally fiber configuration controllable. 
2. Consider the sphere controlled by two independent rotors whose inertia is given by

$$
J=\operatorname{diag}\left(J_{01}, J_{02}, J_{03}\right), \quad M_{12}=\left[\begin{array}{cc}
J_{11} & J_{21} \\
J_{12} & J_{22} \\
J_{13} & J_{23}
\end{array}\right]
$$

If $J_{12} J_{21} \neq J_{11} J_{22}$, then the system is small-time locally fiber configuration controllable and locally fiber equilibrium controllable except in an analytic surface on $S O(3) \times \mathbb{R}^{2}$ of dimension at most four.

3. If the sphere is controlled by three independent rotors, then the system is small-time locally fiber configuration controllable and locally fiber equilibrium controllable at all configurations.

Proof

We consider the three cases as follows:

Case 1: One rotor. Since the reduced dynamics is not locally configuration accessible and the system is real analytic, the complete system is not small-time locally (fiber) configuration controllable.

Case 2: Two independent rotors. With the given inertia $J$ and $M_{12}$, we have

$$
\begin{aligned}
M_{11}(\Gamma)= & J-m_{T} a^{2} \widehat{\Gamma}^{2} \\
= & {\left[\begin{array}{ccc}
J_{01}+m_{T} a\left(\Gamma_{2}^{2}+\Gamma_{3}^{2}\right) & -m_{T} a^{2} \Gamma_{1} \Gamma_{2} & -m_{T} a^{2} \Gamma_{1} \Gamma_{3} \\
-m_{T} a^{2} \Gamma_{1} \Gamma_{2} & J_{02}+m_{T} a\left(\Gamma_{1}^{2}+\Gamma_{3}^{2}\right) & -m_{T} a^{2} \Gamma_{2} \Gamma_{3} \\
-m_{T} a^{2} \Gamma_{1} \Gamma_{3} & -m_{T} a^{2} \Gamma_{2} \Gamma_{3} & J_{03}+m_{T} a\left(\Gamma_{1}^{2}+\Gamma_{2}^{2}\right)
\end{array}\right] }
\end{aligned}
$$

We compute three Lie brackets $\left[\bar{Y}_{1}^{A}, \bar{Y}_{2}^{A}\right],\left[\bar{Y}_{1}^{A},\left[\bar{Y}_{1}^{A}, \bar{Y}_{2}^{A}\right]\right],\left[\bar{Y}_{2}^{A},\left[\bar{Y}_{1}^{A}, \bar{Y}_{2}^{A}\right]\right]$ using the symbolic computation tool Mathematica. Their lengthy expressions are omitted. It can be shown by tedious computations that if $J_{12} J_{21} \neq J_{11} J_{22}$, then the system is locally fiber configuration controllable at the equilibrium $R_{e}=I_{3}$. See the details in the Appendix. Note that each Lie bracket is a real analytic function of $\Gamma$, and the rank condition is expressed in terms of a determinant function of the matrix formed by these Lie brackets, which is also a real analytic function of $\Gamma$. Recall that if a real analytic function on a smooth manifold is nonzero at a point, then it is nonzero on the entire manifold except in an analytic surface with dimension lower than that of the manifold. Using this fact, we conclude that the determinant function is nonzero for all $\Gamma \in \mathbb{S}^{2}$ except in an analytic surface on $\mathbb{S}^{2}$ with dimension less than two. This implies that if $J_{12} J_{21} \neq J_{11} J_{22}$, then the system is locally fiber configuration accessible/controllable at almost all states in $S O(3) \times \mathbb{R}^{2}$ except in an analytic surface on $S O(3) \times \mathbb{R}^{2}$ of dimension at most four.

Case 3: Three independent rotors. We assume that three rotors are independent so that $\operatorname{rank}\left(M_{12}\right)=3$. Therefore, the control vector fields for the reduced dynamics can be chosen as (via some control transformation)

$$
\tilde{Y}_{i}=\left[\begin{array}{c}
e_{i} \\
-M_{12}^{-1} M_{11}(\Gamma) e_{i}
\end{array}\right]=M^{-1}(\Gamma)\left[\begin{array}{c}
0 \\
-\Delta(\Gamma) M_{12}^{-1} M_{11}(\Gamma) e_{i}
\end{array}\right], \quad i=1,2,3
$$


where $\Delta=M_{22}-M_{21} M_{11}^{-1} M_{12}$. Hence, the control vector fields $\bar{Y}_{i}$ for the complete system are isomorphic to

$$
\left[\begin{array}{c}
e_{i} \\
-M_{12}^{-1} M_{11}(\Gamma) e_{i} \\
\Gamma \times e_{i}
\end{array}\right], \quad \text { i.e. } \bar{Y}_{i} \simeq\left[\begin{array}{c}
e_{i} \\
-M_{12}^{-1} M_{11}(\Gamma) e_{i} \\
\Gamma \times e_{i}
\end{array}\right], \quad i=1,2,3
$$

Using the Lie bracket formula (23), we obtain the isomorphisms of the three Lie brackets as

$$
\left[\bar{Y}_{1}, \bar{Y}_{2}\right] \simeq\left[\begin{array}{c}
e_{3} \\
\star \\
\Gamma \times 2 e_{3}
\end{array}\right], \quad\left[\bar{Y}_{1}, \bar{Y}_{3}\right] \simeq\left[\begin{array}{c}
-e_{2} \\
\star \\
-\Gamma \times 2 e_{2}
\end{array}\right], \quad\left[\bar{Y}_{2}, \bar{Y}_{3}\right] \simeq\left[\begin{array}{c}
e_{1} \\
\star \\
\Gamma \times 2 e_{1}
\end{array}\right]
$$

where $\star$ denotes some functions we are not interested in. Therefore, $\left\{\bar{Y}_{1}, \bar{Y}_{2}, \bar{Y}_{3},\left[\bar{Y}_{1}, \bar{Y}_{2}\right]\right.$, $\left.\left[\bar{Y}_{1}, \bar{Y}_{3}\right]\right\}$ span the tangent space of the fiber configuration at any configuration. This implies that the system is locally fiber configuration accessible.

The above results may be obtained from the simplified kinematic model based on the conserved momentum $\Pi$. Suppose the system is at an equilibrium initially. Then $\Pi(t)=0 \forall t \geqslant 0$. Therefore, we obtain $\omega=-A(\Gamma) \dot{r}$, where $A(\Gamma)=M_{11}^{-1}(\Gamma) M_{12}(\Gamma)$. Treating $\dot{r}$ as a control input $v$, we obtain the following kinematic system on $S O(3) \times \mathbb{R}^{3}: \omega=-A(\Gamma) v, \dot{R}=R \widehat{\omega}, \dot{x}=-a e_{3} \times R \omega$, where $\Gamma=R^{\mathrm{T}} e_{3}$. Consequently, one can use this model to obtain the same controllability results as those obtained from the dynamic model. Note that this is not the case for the Chapylygin's top discussed below.

5.2.2. Chaplygin's top. In this case, we assume that $\rho_{c} \neq 0$. Hence, the gravitational force influences the dynamics. The Euler-Poincaré equation is

$$
\frac{\mathrm{d}}{\mathrm{d} t}\left(\frac{\partial l_{c}}{\partial \omega}\right)-\frac{\partial l_{c}}{\partial \omega} \times \omega=m_{T} a_{g} \Gamma \times \rho_{c}
$$

and the shape equation has the same form as (25) (but $l_{c}$ is different). The equilibrium manifold is a one-parameter subgroup of $S O$ (3) for arbitrary rotor angles, that is, $\left\{(R, r) \mid R^{\mathrm{T}} e_{3} \times \rho_{c}=0 \forall r \in Q_{s}\right\}$. This implies that $\Gamma_{e}= \pm \rho_{c} /\left\|\rho_{c}\right\|$. The potential vector field grad $V$ and the control vector fields $Y_{i}^{A}$ are given in (13) and (12), respectively. Since $\partial \rho_{c} / \partial r \equiv 0$, the term in the symmetric product formula (15) becomes

$$
\tilde{\rho}^{*}(\widetilde{X}, \widetilde{Z})+\tilde{\rho}^{*}(\widetilde{Z}, \widetilde{X})=-m_{T} a\left(\left[\left(\rho_{c} \times \Gamma\right) \cdot \omega_{Z}\right] \omega_{X}+\left[\left(\rho_{c} \times \Gamma\right) \cdot \omega_{X}\right] \omega_{Z}\right)
$$

which is zero when evaluated at an equilibrium.

We first look at the case where one rotor is used. In this case, we have

$$
\left\langle Y_{1}^{A}: Y_{1}^{A}\right\rangle^{\sim}=m_{T} a M^{-1}(\Gamma)\left[\begin{array}{c}
2\left[\left(\rho_{c} \times \Gamma\right) \cdot A_{1}(\Gamma)\right] A_{1}(\Gamma) \\
\star
\end{array}\right]
$$


where $\star$ denotes the terms in $T^{*} Q_{s}$. At an equilibrium,

$$
\left\langle Y_{1}^{A}: Y_{1}^{A}\right\rangle^{\sim}\left(\Gamma_{e}\right)=-m_{T} a M^{-1}\left(\Gamma_{e}\right)\left[\begin{array}{l}
0 \\
\star
\end{array}\right]
$$

Hence, the necessary condition for STLCC of the single-input system is satisfied (see Lemma A.1 in the Appendix). However, tedious computations, which are omitted here, show that this system does not generically satisfy the sufficient conditions for local configuration accessibility. Despite this undecidability, we can give the controllability results for the top with two or three rotors as follows.

\section{Proposition 9}

Consider Chaplygin's top controlled by rotors.

1. Suppose the top is controlled by two independent rotors. If the system is locally fiber configuration accessible and satisfies the following condition:

$\left\{M_{12}(1), M_{12}(2), J \Gamma_{e}\right\}$ are linearly independent and $\left(J^{-1} \Gamma_{e}\right) \times\left[M_{12}(1) \times M_{12}(2)\right] \neq 0$

where $M_{12}(1)$ and $M_{12}(2)$ are the first and second columns of $M_{12}$, respectively, then it is small-time locally fiber configuration controllable and locally fiber equilibrium controllable at the equilibrium and the equilibrium manifold.

2. Suppose the top is controlled by three independent rotors. The system is small-time locally fiber configuration controllable and locally fiber equilibrium controllable on the equilibrium manifold.

Before proceeding with the proof of this result, we present a technical lemma essential for the two-rotor case whose proof can be found in the Appendix.

\section{Lemma 10}

If condition (26) is satisfied for the top with two rotors, then the good-bad symmetric product condition holds at the equilibrium.

\section{The Proof of Proposition 9}

Case 1: Two rotors. If the system is locally fiber configuration accessible at an equilibrium, we only need to check the good-bad symmetric product condition on $S O(3) \times Q_{s}$, but the latter follows directly from Lemma 10.

Case 2: Three rotors. Following the similar analysis for the three-rotor case of Chaplygin's sphere, we can show local fiber configuration accessibility at an equilibrium. Thus, it suffices to show the good-bad symmetric product condition. By the argument similar to the proof of Lemma 10, we can verify that bad symmetric products of degrees two and three can be expressed as a linear combination of lower degree good symmetric products at equilibrium. Then, we consider two cases: (1) $\Gamma_{e}$ is not collinear with any $A_{i}\left(\Gamma_{e}\right), i=1,2,3$; and (2) $\Gamma_{e}$ is collinear with only one of $A_{i}\left(\Gamma_{e}\right), i=1,2,3$. Since $\left\{A_{1}(\Gamma), A_{2}(\Gamma), A_{3}(\Gamma)\right\}$ are linearly independent, this classification includes all possible cases. In the first case, we have $\left[\Gamma_{e} \cdot A_{i}\left(\Gamma_{e}\right)\right]^{2} \neq\left\|A_{i}\left(\Gamma_{e}\right)\right\|^{2}, i=1,2,3$. Therefore, any (bad) symmetric products of degree higher than three can be expressed as a linear combination of $Y_{i}^{A},\left\langle\left\langle Y_{i}^{A}: Y_{i}^{A}\right\rangle: Y_{i}^{A}\right\rangle, i=1,2,3$ when evaluated at equilibrium, as shown in the proof of Lemma 10. For the second case, without loss of generality, we may assume that $\Gamma_{e}$ is collinear with $A_{3}\left(\Gamma_{e}\right)$ only. Moreover, it is clear that if $\Gamma_{e} \cdot A_{i}\left(\Gamma_{e}\right) \neq 0$ for $i=1$ or $i=2$, then any 
(bad) symmetric products of degree higher than three can be expressed as a linear combination of $Y_{i}^{A}, i=1,2,3,\left\langle\operatorname{grad} V: Y_{i}^{A}\right\rangle$, and $\left\langle\left\langle Y_{i}^{A}: Y_{i}^{A}\right\rangle: Y_{i}^{A}\right\rangle, i=1,2$, at equilibrium. Suppose $\Gamma_{e} \cdot A_{i}\left(\Gamma_{e}\right)=0$ for $i=1,2$. Then

$$
\left\langle\left\langle\operatorname{grad} V: Y_{1}^{A}\right\rangle: Y_{2}^{A}\right\rangle^{\sim}\left(\Gamma_{e}\right)=m_{T} a_{g} M^{-1}\left(\Gamma_{e}\right)\left[\begin{array}{c}
\Gamma_{e} \times p+\beta\left(\Gamma_{e} \cdot\left[A_{1}\left(\Gamma_{e}\right) \times A_{2}\left(\Gamma_{e}\right)\right]\right) \Gamma_{e} \\
\star
\end{array}\right]
$$

where $p$ is a suitable vector-valued function and $\beta \neq 0$ is a real coefficient. Note that $\Gamma_{e} \times p \in \operatorname{span}$ $\left\{A_{1}\left(\Gamma_{e}\right), A_{2}\left(\Gamma_{e}\right)\right\}$ and $\Gamma_{e} \cdot\left[A_{1}\left(\Gamma_{e}\right) \times A_{2}\left(\Gamma_{e}\right)\right] \neq 0$. This implies that any (bad) symmetric products of degree higher than three can be expressed as a linear combination of $Y_{i}^{A}, i=1,2,3,\langle\langle\operatorname{grad} V$ : $\left.\left.Y_{1}^{A}\right\rangle: Y_{2}^{A}\right\rangle$, and $\left\langle\left\langle Y_{i}^{A}: Y_{i}^{A}\right\rangle: Y_{i}^{A}\right\rangle, i=1,2$, at equilibrium. Consequently, the good-bad symmetric product condition is satisfied.

Moreover, the symmetry property of the equilibrium manifold implies that if the above controllability conditions are satisfied at one equilibrium, then they are also satisfied on the entire equilibrium manifold.

\subsection{Discussions on Chaplygin's top controlled by sliders}

We study another case: a spherical robot controlled by $n$ sliders, see Figure 3 for its schematic configuration.

In this case, the shape configuration is $Q_{s}=\mathbb{R}^{n}$, ignoring sliders' stroke limits. It is clear that the inertia tensor $\bar{M}$ and the position vector $\rho_{c}$ are both functions of shape. Hence, $M$ and $A$ are functions of $(\Gamma, r)$.

Each slider consists of an ideal mass particle that can be translated along the linear axis of the actuator by a motor. Let $v_{i}$ denote a unit vector that defines the axis of the $i$ th slider and let $r_{i}$ denote the relative distance along the axis; let $\rho_{i 0}$ denote the constant position vector from the origin of the base body frame (i.e. the center of the sphere) to the location of the $i$ th slider's axis corresponding to zero $r_{i}$. Hence, the $i$ th slider's position in the base body frame is given by $\rho_{i}(r)=\rho_{i 0}+r_{i} v_{i}, i=1, \ldots, n$. Note $J_{i} \equiv 0, i=1, \ldots, n$. Substituting $\rho_{i}(r)$ 's expression into the inertia tensor matrix $\bar{M}(r)$, we obtain

$$
J(r)=J_{B}-\sum_{i=1}^{n} m_{i} \widehat{\rho}_{i}(r) \widehat{\rho}_{i}(r), \quad J_{B}=J_{0}-m_{0} \widehat{\rho}_{0} \widehat{\rho}_{0}, \quad B_{t}(r)=\left[m_{1} v_{1}, \ldots, m_{n} v_{n}\right]
$$



Figure 3. Schematic configuration of a spherical robot with three sliders. 
$B_{r}(r)=\left[m_{1}\left(\rho_{10} \times v_{1}\right), \ldots, m_{n}\left(\rho_{n 0} \times v_{n}\right)\right]$, and $m(r)=\operatorname{diag}\left\{m_{1}, \ldots, m_{n}\right\}$. Hence, $B_{t}, B_{r}$ and $m$ are all constant. Using these results, we further obtain the expressions for elements in $M(\Gamma, r)$ :

$$
\begin{aligned}
M_{11}(\Gamma, r) & =J_{B}-\sum_{i=1}^{n} m_{i}\left[\rho_{i}(r)+a \Gamma\right]^{\wedge}\left[\rho_{i}(r)+a \Gamma\right]^{\wedge} \\
M_{12}(\Gamma) & =\left[m_{1}\left\{\left(\rho_{10}+a \Gamma\right) \times v_{1}\right\}, \ldots, m_{n}\left\{\left(\rho_{n 0}+a \Gamma\right) \times v_{n}\right\}\right]
\end{aligned}
$$

and $M_{22}=\operatorname{diag}\left\{m_{1}, \ldots, m_{n}\right\}$. For the reduced dynamics, the equilibrium manifold is given by $\left\{\left(R_{e}, r_{e}\right) \mid \Gamma_{e} \times \rho_{c}\left(r_{e}\right)=0\right\}$, where $\Gamma_{e}=R_{e}^{\mathrm{T}} e_{3}$.

Let the constant matrix $E=m_{T}\left(\partial \rho_{c}(r) / \partial r\right)=\left[m_{1} v_{1}, \ldots, m_{n} v_{n}\right]$. It is easy to verify that the following holds in the symmetric product formula (19):

$$
\begin{aligned}
\tilde{\rho}^{*}(\widetilde{X}, \widetilde{Z})+\tilde{\rho}^{*}(\widetilde{Z}, \widetilde{X})= & a\left\{\left[\left(E v_{Z}\right) \cdot \omega_{X}+\left(E v_{X}\right) \cdot \omega_{Z}\right] \Gamma-\left[\left(\rho_{c}(r) \times \Gamma\right) \cdot \omega_{Z}+\left(E v_{Z}\right) \cdot \Gamma\right] \omega_{X}\right. \\
& \left.-\left[\left(\rho_{c}(r) \times \Gamma\right) \cdot \omega_{X}+\left(E v_{X}\right) \cdot \Gamma\right] \omega_{Z}\right\}
\end{aligned}
$$

The control vector fields and the gravitational vector field are given in (12) and (13). Some useful symmetric products evaluated at equilibrium are

$$
\begin{aligned}
& \left\langle Y_{i}^{A}: Y_{j}^{A}\right\rangle^{\sim}=a M^{-1}\left(\Gamma_{e}, r\right) \\
& \quad \times\left[\begin{array}{c}
\left.-\left[m_{i} v_{i} \cdot A_{j}\left(\Gamma_{e}, r\right)+m_{j} v_{j} \cdot A_{i}\left(\Gamma_{e}, r\right)\right] \Gamma_{e}+m_{j}\left(v_{j} \cdot \Gamma_{e}\right) A_{i}\left(\Gamma_{e}, r\right)+m_{i}\left(v_{i} \cdot \Gamma_{e}\right) A_{j}\left(\Gamma_{e}, r\right)\right] \\
\star
\end{array}\right]
\end{aligned}
$$

where $\star$ denotes the term of no interest, and

$$
\begin{aligned}
& \left\langle\operatorname{grad} V: Y_{i}^{A}\right\rangle^{\sim}=-a M^{-1}\left(\Gamma_{e}, r\right)\left[\begin{array}{c}
\Gamma_{e} \times\left[m_{T} \rho_{c}(r) \times A_{i}\left(\Gamma_{e}, r\right)+m_{i} v_{i}\right] \\
0
\end{array}\right] \\
& \left\langle\operatorname{grad} V:\left\langle\operatorname{grad} V: Y_{i}^{A}\right\rangle\right\rangle^{\sim}=-a M^{-1}\left(\Gamma_{e}, r\right) \\
& \quad \times\left[\begin{array}{c}
\Gamma_{e} \times\left\{m_{T} \rho_{c}(r) \times\left[a \tilde{M}_{11}\left(\Gamma_{e}, r\right) \Gamma_{e} \times\left(m_{T} \rho_{c}(r) \times A_{i}\left(\Gamma_{e}, r\right)+m_{i} v_{i}\right)\right]\right. \\
\left.+E\left[-a \tilde{M}_{12}\left(\Gamma_{e}, r\right) \Gamma_{e} \times\left(m_{T} \rho_{c}(r) \times A_{i}\left(\Gamma_{e}, r\right)+m_{i} v_{i}\right)\right]\right\} \\
0
\end{array}\right]
\end{aligned}
$$

where $i, j=1, \ldots, n$. In fact, let

$$
\left(\operatorname{sy}_{\operatorname{grad} V}^{k} Y_{i}^{A}\right)^{\sim}=\left[\begin{array}{c}
\omega_{k}\left(\Gamma_{e}, r\right) \\
v_{k}\left(\Gamma_{e}, r\right)
\end{array}\right]
$$

for $k \in \mathbb{Z}^{+}$, then

$$
\left(\operatorname{sy}_{\operatorname{grad} V}^{k+1} Y_{i}^{A}\right)^{\sim}=-a M^{-1}\left(\Gamma_{e}, r\right)\left[\begin{array}{c}
\Gamma_{e} \times\left[m_{T} \rho_{c}(r) \times\left(-\omega_{k}\left(\Gamma_{e}, r\right)\right)+E v_{k}\left(\Gamma_{e}, r\right)\right] \\
0
\end{array}\right]
$$


Hence, the bad symmetric products of degree two evaluated at the equilibrium are

$$
\left\langle Y_{i}^{A}: Y_{i}^{A}\right\rangle^{\sim}=-a M^{-1}\left(\Gamma_{e}, r\right)\left[\begin{array}{c}
2 m_{i}\left(\left[v_{i} \cdot \Gamma_{e}\right] A_{i}\left(\Gamma_{e}, r\right)-\left[v_{i} \cdot A_{i}\left(\Gamma_{e}, r\right)\right] \Gamma_{e}\right) \\
\star
\end{array}\right], \quad i=1, \ldots, n
$$

Case 1: One slider. The necessary condition for STLCC of the single-input system (given in the Appendix) requires that $\left[\left(v \cdot \Gamma_{e}\right) A_{e}-\left(v \cdot A_{e}\right) \Gamma_{e}\right]$ and $\Gamma_{e} \times h_{i}$ be linearly dependent, where $h_{i}$ is some suitable vector-valued function. This implies that $\left(v \cdot \Gamma_{e}\right)\left(A_{e} \cdot \Gamma_{e}\right)=v \cdot A_{e}$. Consider an equilibrium $\left(R_{e}, r_{e}\right) \in S O(3) \times \mathbb{R}$. The necessary condition and the equilibrium condition thus imply that the system is small-time locally configuration controllable only if the following three equations hold:

$$
\left(v \cdot \Gamma_{e}\right)\left[A\left(\Gamma_{e}, r_{e}\right) \cdot \Gamma_{e}\right]=v \cdot A\left(\Gamma_{e}, r_{e}\right), \quad \Gamma_{e} \times \rho_{c}\left(r_{e}\right)=0, \quad\left\|\Gamma_{e}\right\|=1
$$

It is clear that the curve(s) on $S O(3) \times \mathbb{R}$ that satisfies the above equations is of dimension at most one. This means that the system is generically not small-time locally configuration controllable at equilibrium.

Case 2: Two or more sliders. The sufficient conditions for STLCC require that $\left[v_{i} \cdot \Gamma_{e}\right] A_{i}\left(\Gamma_{e}, r\right)=$ $\left[v_{i} \cdot A_{i}\left(\Gamma_{e}, r\right)\right] \Gamma_{e}$ for each $i=1, \ldots, n$, and that the union of $\left\{\Gamma_{e} \times\left[m_{T} \rho_{c}(r) \times A_{k}\left(\Gamma_{e}, r\right)+\right.\right.$ $\left.\left.m_{k} v_{k}\right], k=1, \ldots, n\right\}$ and

$$
\begin{aligned}
\{- & {\left[m_{i} v_{i} \cdot A_{j}\left(\Gamma_{e}, r\right)+m_{j} v_{j} \cdot A_{i}\left(\Gamma_{e}, r\right)\right] \Gamma_{e}+m_{j}\left(v_{j} \cdot \Gamma_{e}\right) A_{i}\left(\Gamma_{e}, r\right) } \\
& \left.+m_{i}\left(v_{i} \cdot \Gamma_{e}\right) A_{j}\left(\Gamma_{e}, r\right), i \neq j, i, j=1, \ldots, n\right\}
\end{aligned}
$$

span $\mathbb{R}^{3}$ at the equilibrium, as well as local configuration accessibility conditions at this equilibrium. Note that the condition $\left[v_{i} \cdot \Gamma_{e}\right] A_{i}\left(\Gamma_{e}, r_{e}\right)=\left[v_{i} \cdot A_{i}\left(\Gamma_{e}, r_{e}\right)\right] \Gamma_{e} \forall i=1, \ldots, n$ implies that $A_{i}\left(\Gamma_{e}, r_{e}\right)$ is collinear with $\Gamma_{e}$ for all $i=1, \ldots, n$, which does not hold if any two columns of $M_{12}\left(\Gamma_{e}\right)$ are linearly independent. Thus, the sufficient conditions fail to show STLCC and local equilibrium controllability in such a situation.

These results suggest that the spherical robot model is more difficult to control via sliders in 'short time' by small maneuvers. Note that the long-time controllability may be achieved using sliders, see [10].

\section{MOTION PLANNING FOR CHAPLYGIN'S SPHERE AND TOP USING ROTORS}

In this section, we develop motion planning algorithms for Chaplygin's sphere and top controlled by rotors. Perturbation techniques are employed to derive motion planning schemes to achieve pure rotational and pure translational maneuvers of the spherical body. In the following, we use $\bar{X}(q, t)$ to denote its time integral of a time-varying function $X(q, t)$, that is $\bar{X}(q, t)=\int_{0}^{t} X(q, \tau) \mathrm{d} \tau$.

\subsection{Motion planning for Chaplygin's sphere using rotors}

We look at Chaplygin's sphere controlled by $n$ rotors. In this case, the center of mass of the system coincides with the center of the sphere so that there is no gravity acting on the system. Sub-system 
(24) $-(25)$ on $S O(3) \times Q_{s}$ is

$$
M(\Gamma)\left[\begin{array}{c}
\dot{\omega} \\
\ddot{r}
\end{array}\right]=\left[\begin{array}{c}
m_{T} a^{2}(\Gamma \cdot \omega) \Gamma \times \omega+\frac{\partial l_{c}}{\partial \omega} \times \omega \\
0
\end{array}\right]+\left[\begin{array}{c}
0 \\
u_{s}
\end{array}\right]
$$

or equivalently

$$
\left[\begin{array}{c}
\dot{\omega} \\
\ddot{r}
\end{array}\right]=M^{-1}(\Gamma)\left[\begin{array}{c}
m_{T} a^{2}(\Gamma \cdot \omega) \Gamma \times \omega+\frac{\partial l_{c}}{\partial \omega} \times \omega \\
0
\end{array}\right]+\left[\begin{array}{c}
-A(\Gamma) \\
I_{n}
\end{array}\right] \Delta^{-1}(\Gamma) u_{s}
$$

Let control $u_{s}=\varepsilon \Delta(\Gamma) \tilde{u}$, where $\tilde{u}$ is new control and $\varepsilon>0$ is a small number, we obtain $Y(\Gamma, t)=\varepsilon \sum_{i=1}^{n} Y_{i}(\Gamma) \tilde{u}_{i}(t)$, where $Y_{i}(\Gamma)$ is control vector field corresponding to $\tilde{u}_{i}$ given by $\tilde{Y}_{i}(\Gamma)=\left[\begin{array}{c}-A_{i}(\Gamma) \\ e_{i}\end{array}\right]$. Following Bullo's series expansion [12]

$$
\left[\begin{array}{c}
\omega(t) \\
\dot{r}(t)
\end{array}\right]=\overline{\widetilde{Y}}(\Gamma, t)-\frac{1}{2} \overline{\langle\bar{Y}: \bar{Y}\rangle^{\sim}}(\Gamma, t)+\frac{1}{2} \overline{\langle\overline{\langle\bar{Y}: \bar{Y}\rangle}: \bar{Y}\rangle^{\sim}}+O\left(\varepsilon^{4}\right)
$$

we have

$$
\left[\begin{array}{c}
\omega(t) \\
\dot{r}(t)
\end{array}\right]=\varepsilon \sum_{i=1}^{n} \tilde{Y}_{i}(\Gamma) \overline{\tilde{u}}_{i}(t)-\varepsilon^{2} \frac{1}{2} \sum_{i, j=1}^{n}\left\langle Y_{i}: Y_{j}\right\rangle^{\sim}(\Gamma) \overline{\overline{\tilde{u}}_{i} \overline{\tilde{u}}_{j}}(t)+O\left(\varepsilon^{3}\right)
$$

where

$$
\left\langle Y_{i}: Y_{j}\right\rangle^{\sim}(\Gamma)=\left[\begin{array}{c}
-A(\Gamma) \\
I_{n}
\end{array}\right] D \Delta_{i j}(\Gamma), \quad D \Delta_{i j}=\Delta^{-1}\left[\frac{\partial \Delta_{i}}{\partial \Gamma}\left(A_{j} \times \Gamma\right)+\frac{\partial \Delta_{j}}{\partial \Gamma}\left(A_{i} \times \Gamma\right)\right]
$$

Expanding the series around the equilibrium $\Gamma_{e}$, we further have [15]

$$
\begin{aligned}
& {\left[\begin{array}{c}
\omega(t) \\
\dot{r}(t)
\end{array}\right]=\varepsilon \sum_{i=1}^{n}\left[\begin{array}{c}
-A_{i e} \\
e_{i}
\end{array}\right] \overline{\tilde{u}}_{i}(t)+\varepsilon^{2} \sum_{i, j=1}^{n}\left\{\left[\begin{array}{c}
\frac{\partial A_{i e}}{\partial \Gamma}\left[\Gamma_{e} \times A_{j e}\right] \overline{\tilde{\tilde{u}}}_{j} \overline{\tilde{u}}_{i}(t) \\
0
\end{array}\right]\right.} \\
& \left.+\left[\begin{array}{c}
-A\left(\Gamma_{e}\right) \\
I_{n}
\end{array}\right] D \Delta_{i j}\left(\Gamma_{e}\right) \overline{\overline{\tilde{u}}_{i} \overline{\tilde{u}}_{j}}(t)\right\}+O\left(\varepsilon^{3}\right)
\end{aligned}
$$

Using another control transformation $\tilde{u}=v-\varepsilon \sum_{i, j=1}^{n} D \Delta_{i j}\left(\Gamma_{e}\right) \bar{v}_{i} \bar{v}_{j}$, where $v=\left(v_{1}, \ldots, v_{n}\right)$ is a control function, we obtain

$$
\omega(t)=\varepsilon \sum_{i=1}^{n}-A_{i e} \bar{v}_{i}(t)+\varepsilon^{2} \sum_{i=1}^{n} \frac{\partial A_{i e}}{\partial \Gamma}\left[\Gamma_{e} \times\left(\sum_{j=1}^{n} A_{j e}\right)\right] \overline{\bar{v}}_{j} \bar{v}_{i}(t)+O\left(\varepsilon^{3}\right), \quad \dot{r}=\varepsilon \sum_{i=1}^{n} e_{i} \bar{v}_{i}(t)+O\left(\varepsilon^{3}\right)
$$

In the following, we determine the control function $v$ (and hence the original control $u_{s}$ ) so as to achieve a maneuver between equilibrium configurations. 
6.1.1. Approximate solutions for sphere configuration. We use classical perturbation technique to obtain approximate solutions that provide us a map between the control function $v_{i}$ and configuration and velocity solutions. Suppose $\omega(t)$ can be written as $\omega(t, \varepsilon)=\sum_{i=1}^{\infty} \varepsilon^{i} \omega_{i}(t)$, where $\omega_{i}(t)$ is the $i$ th order approximation of $\omega$. An approximate solution for $R$ expressed in the exponential coordinates (i.e. $\left.R(t)=R_{e} e^{\widehat{z}(t)}\right)$ is $z(t, \varepsilon)=\sum_{i=1}^{\infty} \varepsilon^{i} z_{i}(t)$, where $z_{1}(t)=\bar{\omega}_{1}(t), z_{2}(t)=\frac{1}{2}{\overline{\omega_{1}} \times \omega_{1}}_{1}(t)+$ $\bar{\omega}_{2}(t)$, and so on. See [15] and the reference therein for more details.

We further obtain a series expansion for $\Gamma=R^{\mathrm{T}} e_{3}$. Since $R(t)=R_{e} \sum_{k=0}^{\infty} \widehat{z}^{k}(t) / k !$, we can write $R$ as

$$
R(t)=R_{e}\left[I+\widehat{z}_{1}(t)+\varepsilon^{2}\left(\widehat{z}_{2}+\frac{\widehat{z}_{1}^{2}}{2 !}\right)(t)+\varepsilon^{3}\left(\widehat{z}_{3}+\frac{\widehat{z}_{1} \widehat{z}_{2}+\widehat{z}_{2} \widehat{z}_{1}}{2 !}+\frac{\widehat{z}_{1}^{3}}{3 !}\right)(t)\right]+O\left(\varepsilon^{4}\right)
$$

Using this result, we have

$$
\begin{aligned}
\Gamma= & \Gamma_{e}+\varepsilon\left(\Gamma_{e} \times z_{1}\right)(t)+\varepsilon^{2}\left(\Gamma_{e} \times z_{2}+\frac{\left(\Gamma_{e} \times z_{1}\right) \times z_{1}}{2 !}\right)(t) \\
& +\varepsilon^{3}\left(\Gamma_{e} \times z_{3}+\frac{\left(\Gamma_{e} \times z_{2}\right) \times z_{1}+\left(\Gamma_{e} \times z_{1}\right) \times z_{2}}{2 !}+\frac{\left[\left(\Gamma_{e} \times z_{1}\right) \times z_{1}\right] \times z_{1}}{3 !}\right)(t)+O\left(\varepsilon^{4}\right)
\end{aligned}
$$

To obtain a series expansion for $x$, we observe

$$
\begin{aligned}
\dot{x}= & -a e_{3} \times R(t) \omega(t) \\
= & -a e_{3} \times R_{e}\left[\varepsilon \omega_{1}(t)+\varepsilon^{2}\left(\omega_{2}+z_{1} \times \omega_{1}\right)(t)\right. \\
& \left.+\varepsilon^{3}\left(\omega_{3}+z_{1} \times \omega_{2}+z_{2} \times \omega_{1}+\frac{z_{1} \times\left(z_{1} \times \omega_{1}\right)}{2 !}\right)(t)+O\left(\varepsilon^{4}\right)\right]
\end{aligned}
$$

Thus, $x(t)=x_{0}+\varepsilon x_{1}(t)+\varepsilon^{2} x_{2}(t)+\varepsilon^{3} x_{3}(t)+O\left(\varepsilon^{4}\right)$, where $x_{1}(t)=-a e_{3} \times R_{e} \bar{\omega}_{1}(t)$ and $x_{2}(t)=$ $-a e_{3} \times R_{e}\left(\bar{\omega}_{2}+\overline{z_{1} \times \omega_{1}}\right)(t)$. Substituting the above results into Equation (27) and equating the orders of $\varepsilon$, we obtain the approximate solutions:

(1) the order of $\varepsilon$ : $\omega_{1}(t)=-\sum_{i=1}^{n} A_{i e} \bar{v}_{i}(t), z_{1}(t)=-\sum_{i=1}^{n} A_{i e} \overline{\bar{v}}_{i}(t)$, and $x_{1}(t)=a e_{3} \times$ $R_{e}\left(\sum_{i=1}^{n} A_{i e} \overline{\bar{v}}_{i}(t)\right)$, where $A_{i e}:=A_{i}\left(\Gamma_{e}\right)$;

(2) the order of $\varepsilon^{2}$ :

$$
\begin{aligned}
\omega_{2}(t)= & \sum_{i=1}^{n} \frac{\partial A_{i e}}{\partial \Gamma}\left[\Gamma_{e} \times\left(\sum_{j=1}^{n} A_{j e}\right)\right] \overline{\bar{v}}_{j} \bar{v}_{i}(t) \\
z_{2}(t)= & \frac{1}{2} \sum_{i, j=1}^{n} A_{i e} \times A_{j e} \overline{\overline{\bar{v}}}_{i} \bar{v}_{j}(t)+\sum_{i=1}^{n} \frac{\partial A_{i e}}{\partial \Gamma}\left[\Gamma_{e} \times\left(\sum_{j=1}^{n} A_{j e}\right)\right] \overline{\bar{v}}_{j} \bar{v}_{i}(t) \\
& \text { and } x_{2}(t)=-a e_{3} \times R_{e}\left(\bar{\omega}_{2}+\overline{z_{1} \times \omega_{1}}\right)(t)=-a e_{3} \times R_{e}\left(z_{2}+\frac{1}{2}{\overline{\omega_{1}} \times \omega_{1}}\right)(t)
\end{aligned}
$$


where $\partial A_{i e} / \partial \Gamma:=\partial A_{i}(\Gamma) /\left.\partial \Gamma\right|_{\Gamma=\Gamma_{e}}$. Since $A_{i}(\Gamma)=M_{11}^{-1}(\Gamma) M_{12} e_{i}$, we have

$$
\frac{\partial A_{i}(\Gamma)}{\partial \Gamma}=\frac{\partial M_{11}^{-1}(\Gamma)}{\partial \Gamma} M_{12}=-M_{11}^{-1}(\Gamma) \frac{\partial M_{11}(\Gamma)}{\partial \Gamma} M_{11}^{-1}(\Gamma) M_{12} e_{i}=-M_{11}^{-1}(\Gamma) \frac{\partial M_{11}(\Gamma)}{\partial \Gamma} A_{i}(\Gamma)
$$

Noticing that $\partial M_{11}(\Gamma) / \partial \Gamma_{i}=-m_{T} a^{2}\left[\widehat{e}_{i} \widehat{\Gamma}+\widehat{\Gamma} \widehat{e}_{i}\right], i=1,2,3$, we have

$$
\begin{aligned}
\frac{\partial A_{i}(\Gamma)}{\partial \Gamma}= & -M_{11}^{-1}(\Gamma)\left[\frac{\partial M_{11}(\Gamma)}{\partial \Gamma_{1}} A_{i}(\Gamma) \frac{\partial M_{11}(\Gamma)}{\partial \Gamma_{2}} A_{i}(\Gamma) \frac{\partial M_{11}(\Gamma)}{\partial \Gamma_{3}} A_{i}(\Gamma)\right] \\
= & m_{T} a^{2} M_{11}^{-1}(\Gamma)\left\{\left[\widehat{e}_{1}\left[\Gamma \times A_{i}(\Gamma)\right] \widehat{e}_{2}\left[\Gamma \times A_{i}(\Gamma)\right] \widehat{e}_{3}\left[\Gamma \times A_{i}(\Gamma)\right]\right]\right. \\
& \left.+\widehat{\Gamma}\left[\widehat{e}_{1} A_{i}(\Gamma) \widehat{e}_{2} A_{i}(\Gamma) \widehat{e}_{3} A_{i}(\Gamma)\right]\right\}
\end{aligned}
$$

Finally, we obtain the following approximation up to the order of $\varepsilon^{2}$ :

$$
\begin{aligned}
\dot{r}= & \varepsilon \sum_{i=1}^{n} e_{i} \bar{v}_{i}(t)+O\left(\varepsilon^{3}\right) \\
\omega(t, \varepsilon)= & -\varepsilon \sum_{i=1}^{n} A_{i e} \bar{v}_{i}(t)+\varepsilon^{2} \sum_{i=1}^{n} \frac{\partial A_{i e}}{\partial \Gamma}\left[\Gamma_{e} \times\left(\sum_{j=1}^{n} A_{j e}\right)\right] \overline{\bar{v}}_{j} \bar{v}_{i}(t)+O\left(\varepsilon^{3}\right) \\
z(t, \varepsilon)= & -\varepsilon \sum_{i=1}^{n} A_{i e} \overline{\bar{v}}_{i}(t)+\varepsilon^{2}\left\{\sum_{1 \leqslant i<j \leqslant n} D A_{i j e} \overline{\overline{\bar{v}}}_{i} \bar{v}_{j}(t)\right. \\
& \left.+\sum_{1 \leqslant j \leqslant i \leqslant n}\left[\frac{1}{2} A_{j e} \times A_{i e}+\frac{\partial A_{j e}}{\partial \Gamma}\left(\Gamma_{e} \times A_{i e}\right)\right] \overline{\bar{v}}_{i} \overline{\bar{v}}_{j}(t)\right\}+O\left(\varepsilon^{3}\right) \\
x(t, \varepsilon)= & -a e_{3} \times R_{e}\left(\varepsilon^{2} \bar{z}_{2}(t, \varepsilon)+\frac{\varepsilon^{2}}{2}\left[\sum_{1 \leqslant i<j \leqslant n} A_{i e} \times A_{j e}\left(\overline{\bar{v}}_{i} \bar{v}_{j}(t)-\overline{\bar{v}}_{j} \bar{v}_{i}(t)\right)\right.\right. \\
& \left.\left.+\sum_{1 \leqslant j \leqslant i \leqslant n} A_{j e} \times A_{i e} \overline{\bar{v}}_{i} \overline{\bar{v}}_{j}(t)\right]\right)+O\left(\varepsilon^{3}\right)
\end{aligned}
$$

where

$$
D A_{i j e}=A_{i e} \times A_{j e}+\frac{\partial A_{j e}}{\partial \Gamma}\left(\Gamma_{e} \times A_{i e}\right)-\frac{\partial A_{i e}}{\partial \Gamma}\left(\Gamma_{e} \times A_{j e}\right)
$$

is the curvature of the connection $A_{i}$. These results will be used for motion planning design in the sequel.

6.1.2. Motion planning for Chaplygin's sphere via three rotors. Consider the Chaplygin's sphere controlled by three independent rotors, i.e. $\operatorname{rank}\left(M_{12}\right)=n=3$. This suggests $A_{i}(\Gamma), i=1,2,3$, are linearly independent for all $\Gamma$. As shown in Section 5.2.1, the sphere configuration, i.e. its attitude 
and planar position, is controllable at equilibrium using the three rotors. The motion planning algorithm to achieve fiber configuration maneuvers is given as follows. This algorithm also leads to the zero-rotor speed approximately at the final time instant.

Let the final attitude and final position of the sphere be given by $\varepsilon z_{f}$ and $\varepsilon x_{f}=\varepsilon\left(x_{f 1}, x_{f 2}, 0\right)$. And we also assume the initial position is at the origin, without loss generality. Define

$$
D A_{e}=\left[\begin{array}{llll}
D A_{12 e} & D A_{13 e} & D A_{23 e}
\end{array}\right], \quad P_{e}=\left[\begin{array}{llll}
A_{1 e} \times A_{2 e} & A_{1 e} \times A_{3 e} & A_{2 e} \times A_{3 e}
\end{array}\right]
$$

and choose $\bar{x}_{f} \in \mathbb{R}^{3}$ to satisfy $x_{f}=-a e_{3} \times R_{e} \bar{x}_{f}$; note that $P_{e}$ is invertible and $\bar{x}_{f}$ is not unique.

Let $[0, T]$ be a given time interval for $T>0$. Using the approximation formula at the end of the last section, we may divide the motion planning task into two sub-tasks: in the first half time interval $\left[0, \frac{T}{2}\right]$, the second-order terms proportional to $\overline{{\overline{v_{i}}}^{(2)} \overline{v_{j}}-{\overline{v_{i}}}^{(2)} \overline{v_{j}}}, i<j$, are used; in the second half time interval $\left[\frac{T}{2}, T\right]$, the first-order terms are used. That is,

1. Find controls $v_{i}(t), t \in\left[0, \frac{T}{2}\right], i=1,2,3$, that satisfy the following boundary conditions:

$$
\bar{v}_{i}\left(\frac{T}{2}\right)=0, \quad \bar{v}_{i}^{(2)}\left(\frac{T}{2}\right)=0, \quad i=1,2,3
$$

where $\bar{v}_{i}^{(2)}=\overline{\bar{v}}_{i}$, and

$$
\left[\overline{{\overline{v_{1}}}^{(2)} \overline{v_{2}}} \overline{{\overline{v_{1}}}^{(2)} \overline{v_{3}}} \overline{{\overline{v_{2}}}^{(2)} \overline{v_{3}}}\right]\left(\frac{T}{2}\right)=P_{e}^{-1}\left(\bar{x}_{f}-z_{f}\right)
$$

where we use the fact that $\overline{{\overline{v_{i}}}^{(2)} \overline{v_{j}}-{\overline{v_{i}}}^{(2)} \overline{v_{j}}}(T / 2)=2 \overline{{\overline{v_{i}}}^{(2)} \overline{v_{j}}}(T / 2)$ when $\bar{v}_{i}^{(2)}(T / 2)=0$.

2. Let $R(T / 2)=R_{e} \exp \left(\left[D A_{e} P_{e}^{-1}\left(\bar{x}_{f}-z_{f}\right)\right]^{\wedge}\right)$. Find controls $v_{i}(t), t \in(T / 2, T], i=1,2,3$, that satisfy the following boundary conditions:

$$
\bar{v}_{i}(T)=0, \quad i=1,2,3, \quad\left[\bar{v}_{1}^{(2)} \bar{v}_{2}^{(2)} \bar{v}_{3}^{(2)}\right](T)=\left.A^{-1}(\Gamma)\right|_{\Gamma=R^{\mathrm{T}}(T / 2) e_{3}}\left[z_{f}-D A_{e} P_{e}^{-1}\left(\bar{x}_{f}-z_{f}\right)\right]
$$

This algorithm leads to $\dot{r}(T)=O\left(\varepsilon^{2}\right), \omega(T)=O\left(\varepsilon^{2}\right), z(T)=z_{f}+O\left(\varepsilon^{2}\right), x(T)=x_{f}+O\left(\varepsilon^{2}\right)$.

We give specific control functions that accomplish the above two sub-tasks. Suppose $T=4 \pi$ such that $T / 2=2 \pi$. Consider the first sub-task. If $\bar{x}_{f}-z_{f}=0$, then we choose $v_{i}(t)=0$. Now look at the case where $\bar{x}_{f}-z_{f} \neq 0$. Without loss of generality, we assume the first element of $P_{e}^{-1}\left(\bar{x}_{f}-z_{f}\right)$ to be nonzero. Consider the following control functions defined on $[0,2 \pi]$ :

$$
\begin{gathered}
v_{1}(t)=a_{1}\left[\sin t-k_{1} \sin \left(k_{1} t\right)\right], \quad v_{2}=a_{2}\left[\cos t-k_{2} \cos \left(k_{2} t\right)\right] \\
v_{3}=a_{31}\left[\sin t-k_{31} \sin \left(k_{31} t\right)\right]+a_{32}\left[\cos t-k_{32} \cos \left(k_{32} t\right)\right]
\end{gathered}
$$

where $k_{1}, k_{2}, k_{31}$, and $k_{32}$ are distinct positive integers greater than one (but $k_{31}$ and $k_{32}$ can be identical), and $a_{1}, a_{2}, a_{31}$, and $a_{32}$ are amplitude coefficients to be determined. It is easy to verify that $\bar{v}_{i}(2 \pi)=0, \bar{v}_{i}^{(2)}(2 \pi)=0, i=1,2,3$, and

$$
\overline{{\overline{v_{1}}}^{(2)} \overline{v_{2}}}(2 \pi)=-a_{1} a_{2} \pi, \quad \overline{{\overline{v_{1}}}^{(2)} \overline{v_{3}}}(2 \pi)=-a_{1} a_{32} \pi, \quad \overline{{\overline{v_{2}}}^{(2)} \overline{v_{3}}}(2 \pi)=a_{2} a_{31} \pi
$$

Note that in the last condition, we have three equations but four variables. This allows us to choose these variables such that an objective function $a_{1}^{2}+a_{2}^{2}+a_{31}^{2}+a_{32}^{2}$ is minimal. This 
function has the physical meaning that the magnitudes of the control functions are minimal. Let $(1 / \pi) P_{e}^{-1}\left(\bar{x}_{f}-z_{f}\right)=\left(v_{1}, v_{2}, v_{3}\right)^{\mathrm{T}}$, it is easy to see that $a_{1}=\left(\left(v_{1}^{2}+v_{2}^{2}\right) v_{1}^{2} /\left(v_{1}^{2}+v_{3}^{2}\right)\right)^{1 / 4}$ results in the minimum. Then, we choose $a_{2}=-v_{1} / a_{1}, a_{32}=-v_{2} / a_{1}, a_{31}=v_{3} / a_{2}$, where $a_{2} \neq 0$, then the boundary conditions are satisfied. In the second sub-task, we may choose

$$
v_{i}(t)=b_{i} \sin t, \quad t \in[2 \pi, 4 \pi], \quad i=1,2,3
$$

It is clear that $\bar{v}_{i}(4 \pi)=0, \bar{v}_{1}^{(2)}(4 \pi)=2 \pi b_{i}$. Therefore, $\left(b_{1}, b_{2}, b_{3}\right)^{\mathrm{T}}=\left.(1 / 2 \pi) A^{-1}(\Gamma)\right|_{\Gamma=R^{\mathrm{T}}(T / 2) e_{3}}$ $\left[z_{f}-D A_{e} P_{e}^{-1}\left(\bar{x}_{f}-z_{f}\right)\right]$ satisfies the boundary conditions.

\section{Example 11}

Consider Chaplygin's sphere controlled by three rotors. The inertia matrices are $J=\operatorname{diag}(5,5,5)$, and

$$
M_{12}=\left[\begin{array}{lll}
1.0 & 0.1 & 0.1 \\
0.1 & 1.0 & 0.1 \\
0.1 & 0.1 & 1.0
\end{array}\right], \quad M_{22}=\operatorname{diag}(1.0,1.0,1.0)
$$

The total mass of the system is $m_{T}=2$ and the sphere's radius is $a=1$. For illustration, we design two maneuvers: pure rotational maneuver without changing the sphere's initial position and pure translational maneuver without changing the sphere's initial attitude. Clearly, any sphere maneuver can be written as a combination of these two maneuvers. In the following, we assume the initial attitude $R(0)=I_{3}$ and initial position $x(0)=0$.

Pure rotational maneuver: In this maneuver, the desired final configuration is given by $z_{f}=(0.006,-0.006,0.006)$ and $x_{f}=(0,0)$, and $T=4 \pi$. We choose $\bar{x}_{f}=(0,0,0)$ and the other control parameters are chosen as

$$
a_{1}=0.296, \quad a_{2}=0.3255, \quad a_{31}=-0.2212, \quad a_{32}=0.1749, \quad k_{1}=4, \quad k_{2}=3, \quad k_{31}=k_{32}=2
$$

and $b_{1}=-0.0115, b_{2}=0.014, b_{3}=-0.0136$. The time responses of the exponential coordinate $z$ and the position $x$ are given in Figures 4 and 5. Simulations show that the final configuration is given by

$$
z(T) \approx(0.0061,-0.0061,0.0057), \quad x(T) \approx 1.0 \times 10^{-3}(-0.1365,-0.9076)
$$

and $\|\omega(T)\|_{\infty}=1.212 \times 10^{-5},\|\dot{r}(T)\|_{\infty}=8.254 \times 10^{-5}$.

Pure translational maneuver: In this maneuver, suppose the desired final configuration is given by $z_{f}=(0,0,0)$ and $x_{f}=(-0.0025,0.0025)$ at $T=4 \pi$. We choose $\bar{x}_{f}=(-0.0025,-0.0025$, $-0.00075)$, and the other control parameters are determined as

$$
\begin{gathered}
a_{1}=a_{2}=0.1333, \quad a_{31}=a_{32}=-0.2455, \quad k_{1}=2, \quad k_{2}=3, \quad k_{31}=k_{32}=4 \\
b_{1}=b_{2}=-0.001742, \quad b_{3}=-0.000726
\end{gathered}
$$

The time responses of the exponential coordinate $z$ and the position $x$ are given in Figures 6 and 7 . Simulations show that the final configuration is given by

$$
z(T) \approx 1.0 \times 10^{-3}(0.0768,-0.1041,-0.0437), \quad x(T) \approx(-0.0027,0.0022)
$$

and $\|\omega(T)\|_{\infty}=6.098 \times 10^{-6},\|\dot{r}(T)\|_{\infty}=4.45 \times 10^{-5}$. 


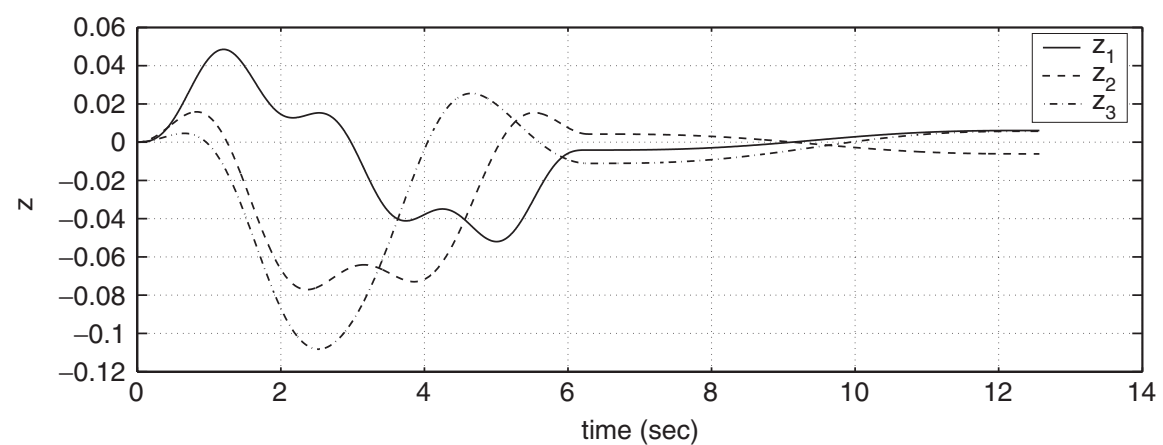

Figure 4. Time response of the exponential coordinate $z$ : pure rotation.

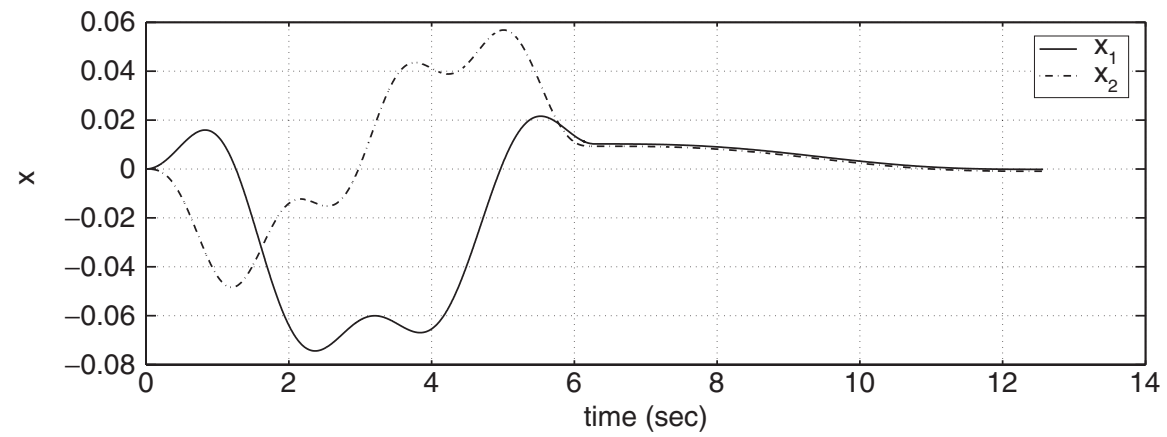

Figure 5. Time response of the sphere position $x$ : pure rotation.



Figure 6. Time response of the exponential coordinate $z$ : pure translation. 


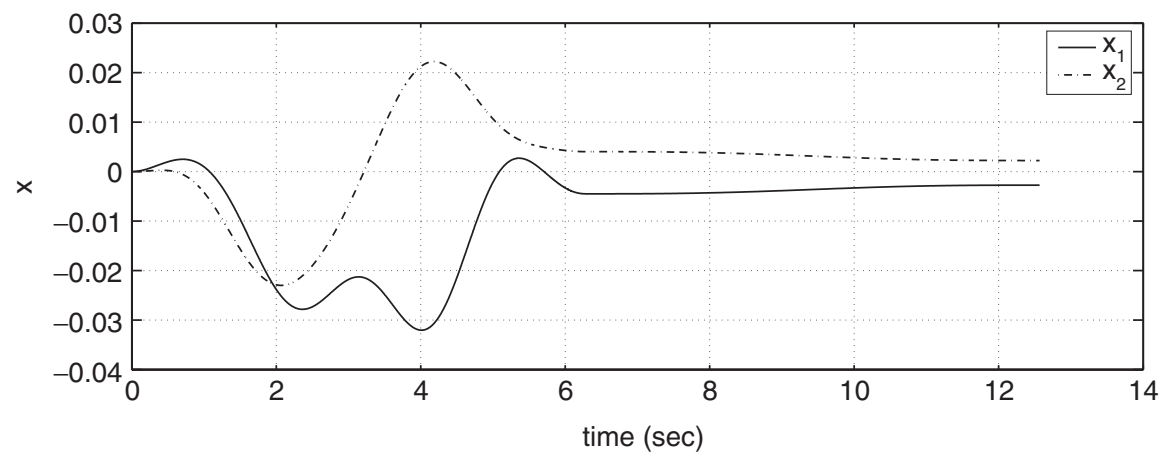

Figure 7. Time response of the sphere position $x$ : pure translation.

\subsection{Motion planning for Chaplygin's top using rotors}

In this case, the reduced equations of motion on $S O(3) \times Q_{s}$ are

$$
\begin{aligned}
M(\Gamma)\left[\begin{array}{c}
\dot{\omega} \\
\ddot{r}
\end{array}\right]= & {\left[\begin{array}{c}
m_{T} a N(\Gamma, \omega)+\frac{\partial l_{c}}{\partial \omega} \times \omega+m_{T} a\left[\left(\rho_{c} \times \Gamma\right) \cdot \omega\right] \omega \\
0
\end{array}\right] } \\
& +\left[\begin{array}{c}
m_{T} a_{g} \Gamma \times \rho_{c} \\
0
\end{array}\right]+\left[\begin{array}{c}
0 \\
u_{s}
\end{array}\right]
\end{aligned}
$$

where $N(\Gamma, \omega)=a(\Gamma \cdot \omega) \Gamma \times \omega-\left(\rho_{c} \times \omega\right) \times(\Gamma \times \omega)-\rho_{c} \times(\omega \times(\Gamma \times \omega))$, or equivalently

$$
\begin{aligned}
{\left[\begin{array}{c}
\dot{\omega} \\
\ddot{r}
\end{array}\right]=} & M^{-1}(\Gamma)\left[\begin{array}{c}
m_{T} a N(\Gamma, \omega)+\frac{\partial l_{c}}{\partial \omega} \times \omega+m_{T} a\left[\left(\rho_{c} \times \Gamma\right) \cdot \omega\right] \omega \\
0
\end{array}\right] \\
& +M^{-1}(\Gamma)\left[\begin{array}{c}
m_{T} a_{g} \Gamma \times \rho_{c} \\
0
\end{array}\right]+\left[\begin{array}{c}
-A(\Gamma) \\
I_{n}
\end{array}\right] \Delta^{-1}(\Gamma) u_{s}
\end{aligned}
$$

where $\rho_{c}$ is constant. Choosing $u_{s}=\Delta(\Gamma) \tilde{u}$, we obtain

$$
\operatorname{grad} V^{\sim}=M^{-1}(\Gamma) m_{T} a_{g}\left[\begin{array}{c}
\Gamma \times \rho_{c} \\
0
\end{array}\right], \quad \tilde{Y}_{i}=\left[\begin{array}{c}
-A_{i} \\
e_{i}
\end{array}\right]=M^{-1}(\Gamma)\left[\begin{array}{c}
0 \\
\Delta_{i}(\Gamma)
\end{array}\right], \quad i=1, \ldots, n
$$

Symmetric product computations show that

$$
\left\langle\operatorname{grad} V: Y_{i}\right\rangle^{\sim}\left(\Gamma_{e}\right)=M^{-1}\left(\Gamma_{e}\right)\left[\begin{array}{c}
\Gamma_{e} \times\left(\rho_{c} \times A_{i e}\right) \\
0
\end{array}\right], \quad\left\langle Y_{i}: Y_{j}\right\rangle^{\sim}\left(\Gamma_{e}\right)=\left[\begin{array}{c}
-A\left(\Gamma_{e}\right) \\
I_{n}
\end{array}\right] D \Delta_{i j e}
$$


Therefore, according to [15], we introduce

$$
\tilde{u}=v-\sum_{i=1}^{n} \tilde{M}_{21}\left(\Gamma_{e}\right)\left[\Gamma_{e} \times\left(\rho_{c} \times A_{i e}\right)\right] \overline{\bar{v}}_{i}+\varepsilon \sum_{i, j=1}^{n} D \Delta_{i j}\left(\Gamma_{e}\right) \bar{v}_{i} \bar{v}_{j}
$$

where $v=\left(v_{1}, \ldots, v_{n}\right)$ is a control function. Thus, we obtain

$$
\begin{aligned}
\omega(t)= & \varepsilon \sum_{i=1}^{n}\left[-A_{i e} \bar{v}_{i}(t)+M_{11}^{-1}\left(\Gamma_{e}\right)\left[\Gamma_{e} \times\left(\rho_{c} \times A_{i e}\right)\right] \bar{v}_{i}^{(3)}(t)\right] \\
& +\varepsilon^{2} \sum_{i=1}^{n} \frac{\partial A_{i e}}{\partial \Gamma}\left[\Gamma_{e} \times\left(\sum_{j=1}^{n} A_{j e}\right)\right] \overline{\bar{v}}_{j} \bar{v}_{i}(t)+O\left(\varepsilon t^{5}\right) \\
\dot{r}= & \varepsilon \sum_{i=1}^{n} e_{i} \bar{v}_{i}(t)+O\left(\varepsilon t^{5}\right)
\end{aligned}
$$

We further have

$$
\begin{aligned}
z(t, \varepsilon)= & \varepsilon \sum_{i=1}^{n}\left[-A_{i e} \overline{\bar{v}}_{i}(t)+M_{11}^{-1}\left(\Gamma_{e}\right)\left[\Gamma_{e} \times\left(\rho_{c} \times A_{i e}\right)\right] \bar{v}_{i}^{(4)}(t)\right]+\varepsilon^{2}\left\{\sum_{1 \leqslant i<j \leqslant n} D A_{i j e} \overline{\overline{\bar{v}}}_{i} \bar{v}_{j}(t)\right. \\
& \left.+\sum_{1 \leqslant j \leqslant i \leqslant n}\left[\frac{1}{2} A_{j e} \times A_{i e}+\frac{\partial A_{j e}}{\partial \Gamma}\left(\Gamma_{e} \times A_{i e}\right)\right] \overline{\bar{v}}_{i} \overline{\bar{v}}_{j}(t)\right\}+O\left(\varepsilon t^{6}\right) \\
x(t, \varepsilon)= & -a e_{3} \times R_{e}\left(\bar{z}_{2}(t, \varepsilon)+\frac{\varepsilon^{2}}{2}\left[\sum_{1 \leqslant i<j \leqslant n} A_{i e} \times A_{j e}\left(\overline{\bar{v}}_{i} \bar{v}_{j}(t)-\overline{\bar{v}}_{j} \bar{v}_{i}(t)\right)\right.\right. \\
& \left.\left.+\sum_{1 \leqslant j \leqslant i \leqslant n} A_{j e} \times A_{i e} \overline{\bar{v}}_{i} \overline{\bar{v}}_{j}(t)\right]\right)+O\left(\varepsilon t^{6}\right)
\end{aligned}
$$

In the following, we assume that the top is controlled by three rotors. Using the above formulas, we obtain a motion planning algorithm similar to that for Chaplygin's sphere:

1. Find $T_{1}$ satisfying $0<T_{1}<T$ and controls $v_{i}(t), t \in\left[0, T_{1}\right], i=1,2,3$ that satisfy the following boundary conditions

$$
\bar{v}_{i}\left(T_{1}\right)=0, \quad \bar{v}_{i}^{(2)}\left(T_{1}\right)=0, \quad \bar{v}_{i}^{(3)}\left(T_{1}\right)=0, \quad \bar{v}_{i}^{(4)}\left(T_{1}\right)=0, \quad i=1,2,3
$$

and

$$
\left[\overline{\bar{v}_{1}^{(2)} \overline{v_{2}}} \overline{{\overline{v_{1}}}^{(2)} \overline{v_{3}}} \overline{{\overline{v_{2}}}^{(2)} \overline{v_{3}}}\right]\left(T_{1}\right)=P_{e}^{-1}\left(\bar{x}_{f}-z_{f}\right)
$$

2. Let $R\left(T_{1}\right)=R_{e} \exp \left(\left[D A_{e} P_{e}^{-1}\left(\bar{x}_{f}-z_{f}\right)\right]^{\wedge}\right)$. Find controls $v_{i}(t), t \in\left(T_{1}, T\right], i=1,2,3$, that satisfy the following boundary conditions:

$$
\bar{v}_{i}(T)=0, \quad i=1,2,3, \quad\left[\bar{v}_{1}^{(2)} \bar{v}_{2}^{(2)} \bar{v}_{3}^{(2)}\right](T)=\left.A^{-1}(\Gamma)\right|_{\Gamma=R^{\mathrm{T}}\left(T_{1}\right) e_{3}}\left[z_{f}-D A_{e} P_{e}^{-1}\left(\bar{x}_{f}-z_{f}\right)\right]
$$


This algorithm leads to $\dot{r}(T)=O\left(\varepsilon T^{3}\right), \omega(T)=O\left(\varepsilon T^{3}\right), z(T)=z_{f}+O\left(\varepsilon T^{4}\right), \quad x(T)=x_{f}+$ $O\left(\varepsilon T^{4}\right)$.

Specific control functions are given to achieve the two sub-objectives. Choose $\omega_{v}$ such that $\omega_{v} T_{1}=2 \pi$. Consider the first sub-task. If $\bar{x}_{f}-z_{f}=0$, we choose $v_{i}(t)=0$. Now look at the case where $\bar{x}_{f}-z_{f} \neq 0$. As before, we assume the first element of $P_{e}^{-1}\left(\bar{x}_{f}-z_{f}\right)$ to be nonzero. Consider the following control functions defined on $\left[0,2 \pi / \omega_{v}\right]$ :

$$
\begin{aligned}
v_{1}(t)= & a_{1} \omega_{v}\left(\left[\sin \left(\omega_{v} t\right)-2^{3} \sin \left(2 \omega_{v} t\right)\right]-\frac{3}{7}\left[3^{3} \sin \left(3 \omega_{v} t\right)-4^{3} \sin \left(4 \omega_{v} t\right)\right]\right) \\
v_{2}(t)= & a_{2} \omega_{v}\left[\cos \left(\omega_{v} t\right)-5^{2} \cos \left(5 \omega_{v} t\right)\right] \\
v_{3}(t)= & a_{31} \omega_{v}\left(\left[\sin \left(\omega_{v} t\right)-3^{3} \sin \left(3 \omega_{v} t\right)\right]-\frac{2}{3}\left[2^{3} \sin \left(2 \omega_{v} t\right)-4^{3} \sin \left(4 \omega_{v} t\right)\right]\right) \\
& +a_{32} \omega_{v}\left[\cos \left(\omega_{v} t\right)-5^{2} \cos \left(5 \omega_{v} t\right)\right]
\end{aligned}
$$

where $a_{1}, a_{2}, a_{31}$, and $a_{32}$ are the amplitude coefficients to be determined. Let $T_{1}=2 \pi / \omega_{v}$, it is easy to verify that $\bar{v}_{i}^{(k)}\left(T_{1}\right)=0, i=1,2,3, k=1,2,3,4$, and

$$
\overline{{\overline{v_{1}}}^{(2)} \overline{v_{2}}}\left(T_{1}\right)=-\frac{a_{1} a_{2} \pi}{\omega_{v}^{2}}, \quad \overline{{\overline{v_{1}}}^{(2)} \overline{v_{3}}}\left(T_{1}\right)=-\frac{a_{1} a_{32} \pi}{\omega_{v}^{2}}, \quad \overline{\bar{v}_{2}^{(2)} \overline{v_{3}}}\left(T_{1}\right)=\frac{a_{2} a_{31} \pi}{\omega_{v}^{2}}
$$

In the second sub-task, we choose

$$
v_{i}(t)=b_{i} \tilde{\omega}_{v} \sin \left(\tilde{\omega}_{v} t\right), \quad t \in\left[\frac{2 \pi}{\omega_{v}}, \frac{2 \pi}{\omega_{v}}+\frac{2 \pi}{\tilde{\omega}_{v}}\right], \quad i=1,2,3
$$

which implies that $T=2 \pi / \omega_{v}+2 \pi / \tilde{\omega}_{v}$. It is clear that $\bar{v}_{1}^{(2)}(T)=\left(2 \pi / \tilde{\omega}_{v}\right) b_{i}$. Therefore, $\left(b_{1}, b_{2}, b_{3}\right)^{\mathrm{T}}=\left.\left(\tilde{\omega}_{v} / 2 \pi\right) A^{-1}(\Gamma)\right|_{\Gamma=R^{\mathrm{T}}(T / 2) e_{3}}\left[z_{f}-D A_{e} P_{e}^{-1}\left(\bar{x}_{f}-z_{f}\right)\right]$ satisfies the boundary conditions.

\section{Example 12}

Consider Chaplygin's top with the same mass and inertia values as those in the previous sphere example. The position vector of the center of mass is given by $\rho_{c}=(0,0,-0.1)$. The equilibrium manifold is characterized by $\left\{(R, r) \mid \Gamma=R^{\mathrm{T}} e_{3}= \pm e_{3} \quad \forall r \in \mathbb{S} \times \mathbb{S} \times \mathbb{S}\right\}$. We look at the pure rotational maneuver in this example; the pure translational maneuver can be constructed in the similar manner. Notice that the final configuration must be in the equilibrium manifold, that is, $R_{f}$ must satisfy $R_{f}^{\mathrm{T}} e_{3}= \pm e_{3}$. For the pure rotational maneuver, this implies that the desired final value of $\Gamma$ is $\pm e_{3}$ or $z_{f}=\lambda e_{3}$ for some $\lambda \in \mathbb{R}$. Therefore, $x_{f}$ is always zero as desired. This observation allows us to simplify motion planning design: the first sub-task can be skipped, and it suffices to consider the second sub-task only to accomplish a pure rotational maneuver. As an example, we assume that the desired final configuration is given by $z_{f}=(0.0,0.0,0.1)$ and $x_{f}=(0,0)$, and $T=0.1$. We choose $\tilde{\omega}_{v}=20 \pi$ and $b_{1}=-0.463, b_{2}=0.463, b_{3}=-5.0925$. The time responses of $z$ and $x$ are given in Figures 8 and 9. Simulations show that the final configuration is given by

$$
z(T) \approx(0.00,0.00,0.10), \quad x(T) \approx 1.0 \times 10^{-17}(-0.1043,-0.0129)
$$

and $\|\omega(T)\|_{\infty}=1.484 \times 10^{-14},\|\dot{r}(T)\|_{\infty}=6.177 \times 10^{-14}$. 


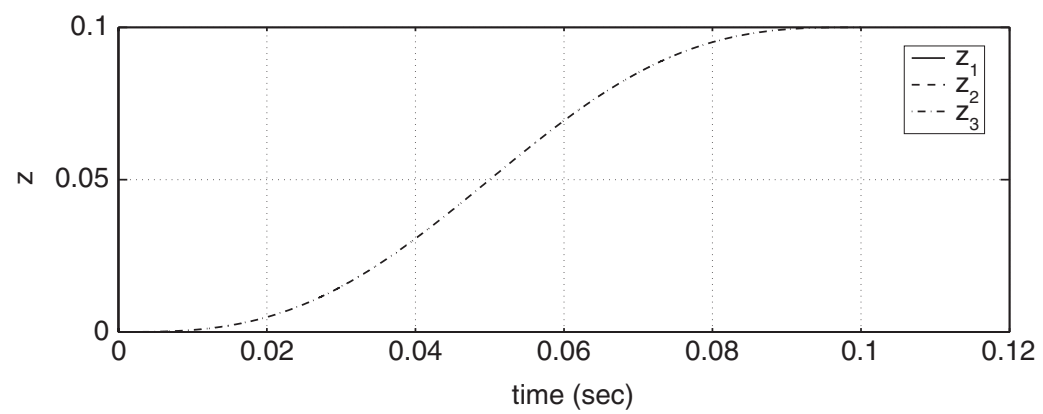

Figure 8 . Time response of the exponential coordinate $z$ : pure rotation.

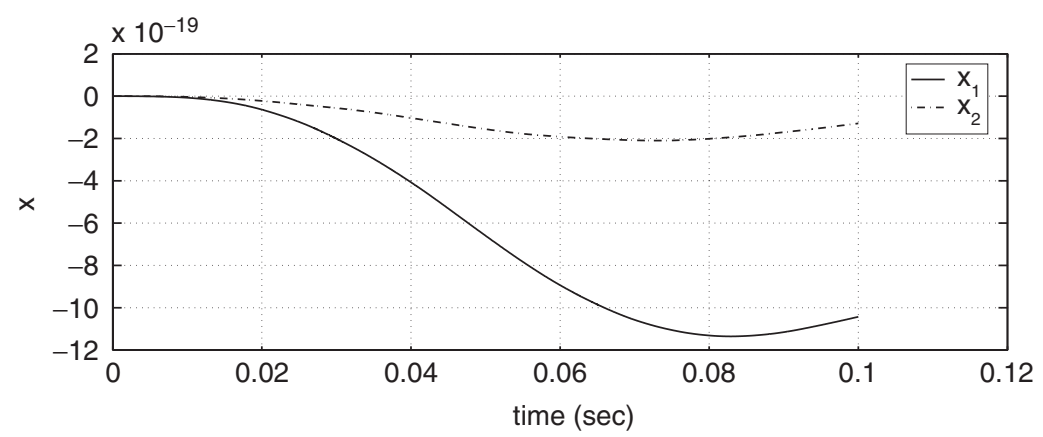

Figure 9. Time response of the sphere position $x$ : pure rotation.

\section{CONCLUSIONS}

Local configuration/equilibrium controllability and motion planning have been studied for a class of constrained multibody systems controlled via internal actuators. The system dynamics depend on an advected parameter and fit the framework of the Euler-Poincare equations. As an example, we consider a controlled multibody spherical robot that can be modeled as a Chaplygin's sphere or a Chaplygin's top. Specific controllability and motion planning results are obtained for the spherical robot controlled by rotors, which illustrate the theory.

\section{APPENDIX A}

A.1. A necessary condition for small-time local configuration controllability of single-input mechanical systems

Consider a single-input simple mechanical system on a manifold $Q$. Let $q=\left(q_{1}, \ldots, q_{n}\right)$ be local coordinates. The equations of motion are

$$
\frac{\mathrm{d}}{\mathrm{d} t}\left[\begin{array}{l}
q \\
\dot{q}
\end{array}\right]=\left[\begin{array}{c}
\dot{q} \\
-\Gamma(q, \dot{q})
\end{array}\right]+\left[\begin{array}{c}
0 \\
Y_{g}(q)
\end{array}\right]+\left[\begin{array}{c}
0 \\
Y_{a}(q)
\end{array}\right] u
$$


where $\Gamma(q, \dot{q})$ has components $\Gamma_{j k}^{i}(q) \dot{q}^{j} \dot{q}^{k}$ (i.e. $\Gamma_{j k}^{i}(q)$ 's are Christoffel symbols), $Y_{g}(q)$ denotes a potential vector field on $Q$, and $Y_{a}(q)$ represents a control vector field on $Q$ for the singlebounded control input $u(t) \in \mathbb{R}$. Furthermore, all the vector fields and components of $\Gamma$ are assumed to be analytic. Using the lift notation for the potential vector field and the control vector field, we denote the vector fields in Equation (A1) as

$$
Z_{g}=\left[\begin{array}{c}
\dot{q} \\
-\Gamma(q, \dot{q})
\end{array}\right], \quad f_{g}=Y_{g}^{\mathrm{lift}}, \quad g_{a}=Y_{a}^{\mathrm{lift}}
$$

We further introduce the following symmetric product operator $\operatorname{sy}_{Y_{g}}^{k} Y_{a}$ for notational convenience:

$$
\operatorname{sy}_{Y_{g}}^{0} Y_{a}=Y_{a}, \quad \operatorname{sy}_{Y_{g}}^{1} Y_{a}=\left\langle Y_{g}: Y_{a}\right\rangle, \quad \operatorname{sy}_{Y_{g}}^{k+1} Y_{a}=\left\langle Y_{g}: \operatorname{sy}_{Y_{g}}^{k} Y_{a}\right\rangle, \quad k \in \mathbb{Z}^{+}
$$

Assume that $q_{e}$ is an equilibrium for system (A1), i.e. $Y_{g}\left(q_{e}\right)=0$. A necessary condition is given as follows.

\section{Lemma 13}

Let $v_{q}=0_{q_{e}}$ be an equilibrium of the mechanical system (A1) where $Y_{g}\left(q_{e}\right)=0$. The system is small-time locally controllable and small-time locally configuration controllable at $0_{q_{e}}$ only if

$$
\left\langle Y_{a}: Y_{a}\right\rangle\left(q_{e}\right) \in \operatorname{span}\left\{Y_{a}\left(q_{e}\right), \operatorname{sy}_{Y_{g}}^{1} Y_{a}\left(q_{e}\right), \ldots, \operatorname{sy}_{Y_{g}}^{n-1} Y_{a}\left(q_{e}\right)\right\}
$$

\section{Proof}

The proof follows from Lewis's result for single-input mechanical systems without potential [21], which is an extension of Sussmann's necessary condition [22] for single-input analytic nonlinear systems. This necessary condition says that a nonlinear system on $x \in \mathbb{R}^{n}$ is small-time locally controllable at an equilibrium $x_{e}$ only if $[g,[f, g]]\left(x_{e}\right) \in \operatorname{span}\left\{f, g, \operatorname{ad}_{f}^{i} g \forall i \in \mathbb{Z}^{+}\right\}\left(x_{e}\right)$, where $f$ and $g$ are drift and control vector fields, respectively.

For system (A1), $f=Z_{g}+f_{g}$ is zero when evaluated at $v_{q}=0_{q_{e}}$. Furthermore, the Lie brackets of the vector fields $Z_{g}, f_{g}, g_{a}$ have special structure. Some useful facts shown in [13] are

$$
\begin{gathered}
{\left[f_{g}, g_{a}\right] \equiv 0, \quad\left[Z_{g}, Y_{a}^{\text {lift }}\right]\left(0_{q_{e}}\right)=-Y_{a}\left(q_{e}\right), \quad\left[X^{\text {lift }},\left[Z_{g}, Y_{a}^{\text {lift }}\right]\right]=\left\langle X: Y_{a}\right\rangle^{\text {lift }}} \\
\operatorname{ad}_{Z_{g}}^{k} X^{\text {lift }}\left(0_{q_{e}}\right)=0, \quad k \geqslant 2
\end{gathered}
$$

where $X$ is a vector field on $Q$. Using these facts, we obtain

$$
\begin{aligned}
& \operatorname{ad}_{f} g_{a}=\left[f, g_{a}\right]=\left[Z_{g}+f_{g}, g_{a}\right]=\left[Z_{g}, g_{a}\right]=\left[Z_{g}, Y_{a}^{\text {lift }}\right] \\
& \operatorname{ad}_{f}^{2} g_{a}=\left[Z_{g}+f_{g},\left[Z_{g}, g_{a}\right]\right]=\operatorname{ad}_{Z_{g}}^{2} g_{a}+\left\langle Y_{g}: Y_{a}\right\rangle^{\text {lift }}
\end{aligned}
$$

where $\operatorname{ad}_{Z_{g}}^{2} g_{a}\left(0_{q_{e}}\right)=0$. Hence, we claim

$$
\operatorname{ad}_{f}^{2 k-1} g_{a}=\left[Z_{g},\left(\operatorname{sy}_{Y_{g}}^{k-1} Y_{a}\right)^{\mathrm{lift}}\right]+\tilde{Y}^{k-1}\left(v_{q}\right), \quad \operatorname{ad}_{f}^{2 k} g_{a}=\left(\operatorname{sy}_{Y_{g}}^{k} Y_{a}\right)^{\mathrm{lift}}+\tilde{Y}^{2 k}\left(v_{q}\right), \quad k \in \mathbb{Z}^{+}
$$

where $\tilde{Y}^{2 k-1}$ and $\tilde{Y}^{2 k}$ are vector fields on $Q$ and become zero when evaluated at $v_{q}=0_{q_{e}}$. 
We show the claim by induction on $k$. For $k=1$, the previous computations have shown the claim holds. Now assume that the claim is true for $1,2, \ldots, k$ where $k \geqslant 1$. We then consider the case of $k+1$, where we need to compute $\operatorname{ad}_{f}^{2 k+1} g_{a}$ and $\operatorname{ad}_{f}^{2(k+1)} g_{a}$. By the induction hypothesis, we have

$$
\begin{aligned}
\operatorname{ad}_{f}^{2 k+1} g_{a} & =\left[Z_{g}+f_{g}, \operatorname{ad}_{f}^{2 k} g_{a}\right]=\left[Z_{g}+Y_{g}^{\mathrm{lift}},\left(\operatorname{sy}_{Y_{g}}^{k} Y_{a}\right)^{\mathrm{lift}}\right]+\left[Z_{g}+Y_{g}^{\mathrm{lift}}, \tilde{Y}^{2 k}\right] \\
& =\left[Z_{g},\left(\operatorname{sy}_{Y_{g}}^{k} Y_{a}\right)^{\mathrm{lift}}\right]+\left[Z_{g}+Y_{g}^{\mathrm{lift}}, \tilde{Y}^{2 k}\right] \\
& =\left[Z_{g},\left(\operatorname{sy}_{Y_{g}}^{k} Y_{a}\right)^{\mathrm{lift}}\right]+\tilde{Y}^{2 k+1}\left(v_{q}\right)
\end{aligned}
$$

where $\tilde{Y}^{2 k+1}\left(v_{q}\right)=\left[Z_{g}+Y_{g}^{\text {lift }}, \tilde{Y}^{2 k}\right]\left(v_{q}\right)$. Note that $Z_{g}\left(0_{q_{e}}\right)=0, Y_{g}^{\text {lift }}\left(0_{q_{e}}\right)=0$ and $\tilde{Y}^{2 k}\left(0_{q_{e}}\right)=0$, thus $\tilde{Y}^{2 k+1}\left(0_{q_{e}}\right)=\left[Z_{g}+Y_{g}^{\text {lift }}, \tilde{Y}^{2 k}\right]\left(0_{q_{e}}\right)=0$, following the local coordinate expression of Lie bracket operation. Similarly,

$$
\begin{aligned}
\operatorname{ad}_{f}^{2(k+1)} g_{a} & =\left[Z_{g}+Y_{g}^{\mathrm{lift}}, \operatorname{ad}_{f}^{2 k+1} g_{a}\right]=\left[Z_{g}+Y_{g}^{\mathrm{lift}},\left[Z_{g},\left(\operatorname{sy}_{Y_{g}}^{k} Y_{a}\right)^{\mathrm{lift}}\right]\right]+\left[Z_{g}+Y_{g}^{\mathrm{lift}}, \tilde{Y}^{2 k+1}\right] \\
& =\left[Y_{g}^{\mathrm{lift}},\left[Z_{g},\left(\operatorname{sy}_{Y_{g}}^{k} Y_{a}\right)^{\mathrm{lift}}\right]\right]+\left[Z_{g},\left[Z_{g},\left(\operatorname{sy}_{Y_{g}}^{k} Y_{a}\right)^{\mathrm{lift}}\right]\right]+\left[Z_{g}+Y_{g}^{\mathrm{lift}}, \tilde{Y}^{2 k+1}\right] \\
& =\left\langle Y_{g}: \operatorname{sy}_{Y_{g}}^{k} Y_{a}\right\rangle^{\mathrm{lift}}+\operatorname{ad}_{Z_{g}}^{2}\left(\operatorname{sy}_{Y_{g}}^{k} Y_{a}\right)^{\mathrm{lift}}+\left[Z_{g}+Y_{g}^{\mathrm{lift}}, \tilde{Y}^{2 k+1}\right] \\
& =\left(\operatorname{sy}_{Y_{g}}^{k+1} Y_{a}\right)^{\mathrm{lift}}+\tilde{Y}^{2(k+1)}\left(v_{q}\right)
\end{aligned}
$$

where $\tilde{Y}^{2(k+1)}\left(v_{q}\right)=\operatorname{ad}_{Z_{g}}^{2}\left(\operatorname{sy}_{Y_{g}}^{k} Y_{a}\right)^{\text {lift }}\left(v_{q}\right)+\left[Z_{g}+Y_{g}^{\text {lift }}, \tilde{Y}^{2 k+1}\right]\left(v_{q}\right)$ is zero when evaluated at $v_{q}=0_{q_{e}}$. This completes the proof of the claim.

We return to the proof of the lemma. Note that

$$
\left[g_{a},\left[f, g_{a}\right]\right]=\left[g_{a},\left[Z_{g}, g_{a}\right]\right]=\left[Y_{a}^{\mathrm{lift}},\left[Z_{g}, Y_{a}^{\mathrm{lift}}\right]\right]=\left\langle Y_{a}: Y_{a}\right\rangle^{\mathrm{lift}}
$$

Evaluating the above brackets at $v_{q}=0_{q_{e}}$ and writing them in matrix form, we have

$$
\begin{gathered}
{\left[g_{a},\left[f, g_{a}\right]\right]\left(0_{q_{e}}\right)=\left[\begin{array}{c}
0 \\
\left\langle Y_{a}: Y_{a}\right\rangle\left(q_{e}\right)
\end{array}\right], \quad \operatorname{ad}_{f}^{2 k-1} g_{a}\left(0_{q_{e}}\right)=\left[\begin{array}{c}
-\operatorname{sy}_{Y_{g}}^{k-1} Y_{a}\left(q_{e}\right) \\
0
\end{array}\right]} \\
\operatorname{ad}_{f}^{2 k} g_{a}\left(0_{q_{e}}\right)=\left[\begin{array}{c}
0 \\
\operatorname{sy}_{Y_{g}}^{k} Y_{a}\left(q_{e}\right)
\end{array}\right], \quad k \in \mathbb{Z}^{+}
\end{gathered}
$$

Consequently, Sussmann's necessary condition is equivalent to

$$
\left\langle Y_{a}: Y_{a}\right\rangle\left(q_{e}\right) \in \operatorname{span}\left\{Y_{g}\left(q_{e}\right), \operatorname{sy}_{Y_{g}}^{k} Y_{a}\left(q_{e}\right), k \in \mathbb{Z}^{+}\right\}
$$

Moreover, since $Y_{g}\left(q_{e}\right)=0$, using the local coordinate expression of symmetric product operation, it is clear that $\left\langle Y_{g}: X\right\rangle\left(q_{e}\right)=\left(\partial Y_{g} / \partial q\right)\left(q_{e}\right) X\left(q_{e}\right)$, where $X$ is a vector field on $Q$. Using this observation and defining $A=\left(\partial Y_{g} / \partial q\right)\left(q_{e}\right)$, we obtain $\operatorname{sy}_{Y_{g}}^{k} Y_{a}\left(q_{e}\right)=A^{k} Y_{a}\left(q_{e}\right), k \in \mathbb{Z}^{+}$. 
By Cayley-Hamilton Theorem, $A^{l} Y_{a}\left(q_{e}\right), l \geqslant n$ is a linear combination of $A^{j} Y_{a}\left(q_{e}\right)$ 's, $0 \leqslant j \leqslant n-1$. Hence, Sussmann's condition is equivalent to

$$
\left\langle Y_{a}: Y_{a}\right\rangle\left(q_{e}\right) \in \operatorname{span}\left\{Y_{g}\left(q_{e}\right), \operatorname{sy}_{Y_{g}} Y_{a}\left(q_{e}\right), \ldots, \operatorname{sy}_{Y_{g}}^{n-1} Y_{a}\left(q_{e}\right)\right\}
$$

Following the argument of [21], we see that this condition is also necessary for STLCC.

\section{A.2. Local configuration accessibility test for Chaplygin's sphere}

This section provides details of Lie bracket computations for local configuration accessibility test for Chaplygin's sphere controlled by two rotors in Section 5.2.1. Define

$$
\Delta_{12}=J_{12} J_{21}-J_{11} J_{22}, \quad \Delta_{13}=J_{13} J_{21}-J_{11} J_{23}, \quad \Delta_{23}=J_{13} J_{22}-J_{12} J_{23}
$$

The vector fields evaluated at $R_{e}=I_{3}$, where $\Gamma_{e}=e_{3}$, are

$$
\bar{Y}_{1}^{A}\left(R_{e}\right) \simeq\left[\begin{array}{c}
-\frac{J_{11}}{J_{01}+m_{T} a^{2}} \\
-\frac{J_{12}}{J_{02}+m_{T} a^{2}} \\
-\frac{J_{13}}{J_{03}} \\
1 \\
0 \\
\frac{a J_{12}}{J_{02}+m_{T} a^{2}} \\
-\frac{a J_{11}}{J_{01}+m_{T} a^{2}} \\
0
\end{array}\right], \quad \bar{Y}_{2}^{A}\left(R_{e}\right) \simeq\left[\begin{array}{c}
-\frac{J_{21}}{J_{01}+m_{T} a^{2}} \\
-\frac{J_{22}}{J_{02}+m_{T} a^{2}} \\
-\frac{J_{23}}{J_{03}} \\
0 \\
1 \\
\frac{a J_{22}}{J_{02}+m_{T} a^{2}} \\
-\frac{a J_{21}}{J_{01}+m_{T} a^{2}} \\
0 \\
{\left[\bar{Y}_{1}^{A}, \bar{Y}_{2}^{A}\right]\left(R_{e}\right) \simeq \frac{1}{\Delta_{1}}} \\
-J_{01} \Delta_{23} \\
J_{02} \Delta_{13} \\
-\left[J_{03}+2 m_{T} a^{2}\right] \Delta_{12} \\
0 \\
0
\end{array}\right]
$$

where $\Delta_{1}=J_{03}\left(J_{01}+m_{T} a^{2}\right)\left(J_{02}+m_{T} a^{2}\right)$,

$$
\left[\bar{Y}_{1}^{A},\left[\bar{Y}_{1}^{A}, \bar{Y}_{2}^{A}\right]\right]\left(R_{e}\right) \simeq \frac{1}{\Delta_{2}}\left[\begin{array}{lllllllll}
\Upsilon_{21} & \Upsilon_{22} & \Upsilon_{23} & 0 & 0 & \Upsilon_{26} & \Upsilon_{27} & 0
\end{array}\right]^{\mathrm{T}}
$$


where $\Delta_{2}=J_{03}^{2}\left(J_{01}+m_{T} a^{2}\right)^{2}\left(J_{02}+m_{T} a^{2}\right)^{2}$ with

$$
\begin{aligned}
\Upsilon_{21}= & -2 m_{T} a^{2} J_{03}^{2} J_{12} \Delta_{12}+J_{01}\left[J_{03} J_{12}\left(J_{03}+3 m_{T} a^{2}\right) \Delta_{12}+J_{02} J_{13}\left(J_{02}+m_{T} a^{2}\right) \Delta_{13}\right] \\
\Upsilon_{22}= & 2 m_{T} a^{2} J_{03}^{2} J_{11} \Delta_{12}+J_{02}\left[-J_{03} J_{11}\left(J_{03}+3 m_{T} a^{2}\right) \Delta_{12}+J_{01} J_{13}\left(J_{01}+m_{T} a^{2}\right) \Delta_{13}\right] \\
\Upsilon_{23}= & -4 m_{T}^{2} a^{4}\left(J_{02} J_{11}+J_{01} J_{12}\right) \Delta_{13}+m_{T}^{2} a^{4} J_{03}\left[J_{13}\left(J_{11} J_{21}+J_{12} J_{22}\right)-J_{23}\left(J_{11}^{2}+J_{12}^{2}\right)\right] \\
& -\left(J_{02}^{2} J_{11}+J_{01}^{2} J_{12}\right)\left[J_{03}+m_{T} a^{2}\right] \Delta_{13} \\
\Upsilon_{26}= & a\left[m_{T} a^{2}\left[J_{03} J_{11}\left(J_{03}-2 m_{T} a^{2}\right) \Delta_{12}+J_{01} J_{13}\left(J_{01}+m_{T} a^{2}\right) \Delta_{23}\right]\right. \\
& \left.+J_{02}\left[-J_{03} J_{11}\left(2 J_{03}+5 m_{T} a^{2}\right) \Delta_{12}+2 J_{01} J_{13}\left(J_{01}+m_{T} a^{2}\right) \Delta_{23}\right]\right] \\
\Upsilon_{27}= & -a\left[m_{T} a^{2}\left[J_{03} J_{12}\left(-J_{03}+2 m_{T} a^{2}\right) \Delta_{12}+J_{02} J_{13}\left(J_{02}+m_{T} a^{2}\right) \Delta_{23}\right]\right. \\
& \left.+J_{01}\left[J_{03} J_{12}\left(2 J_{03}+5 m_{T} a^{2}\right) \Delta_{12}+2 J_{02} J_{13}\left(J_{02}+m_{T} a^{2}\right) \Delta_{23}\right]\right]
\end{aligned}
$$

and

$$
\left[\bar{Y}_{2}^{A},\left[\bar{Y}_{1}^{A}, \bar{Y}_{2}^{A}\right]\right]\left(R_{e}\right) \simeq \frac{1}{\Delta_{3}}\left[\begin{array}{llllllll}
\Upsilon_{31} & \Upsilon_{32} & \Upsilon_{33} & 0 & 0 & \Upsilon_{36} & \Upsilon_{37} & 0
\end{array}\right]^{\mathrm{T}}
$$

where $\Delta_{3}=J_{03}^{2}\left(J_{01}+m_{T} a^{2}\right)^{2}\left(J_{02}+m_{T} a^{2}\right)^{2}$ with

$$
\begin{aligned}
\Upsilon_{31}= & -2 m_{T} a^{2} J_{03}^{2} J_{22} \Delta_{12}+J_{01}\left[J_{03} J_{22}\left(J_{03}+3 m_{T} a^{2}\right) \Delta_{12}+J_{02} J_{23}\left(J_{02}+m_{T} a^{2}\right) \Delta_{13}\right] \\
\Upsilon_{32}= & 2 m_{T} a^{2} J_{03}^{2} J_{21} \Delta_{12}+J_{02}\left[-J_{03} J_{21}\left(J_{03}+3 m_{T} a^{2}\right) \Delta_{12}+J_{01} J_{23}\left(J_{01}+m_{T} a^{2}\right) \Delta_{13}\right] \\
\Upsilon_{33}= & -4 m_{T}^{2} a^{4}\left(J_{02} J_{21}+J_{01} J_{22}\right) \Delta_{13}+m_{T}^{2} a^{4} J_{03}\left[-J_{23}\left(J_{11} J_{21}+J_{12} J_{22}\right)+J_{13}\left(J_{21}^{2}+J_{22}^{2}\right)\right] \\
& -\left(J_{02}^{2} J_{21}+J_{01}^{2} J_{22}\right)\left[J_{03}+m_{T} a^{2}\right] \Delta_{13} \\
\Upsilon_{36}= & a\left[m_{T} a^{2}\left[J_{03} J_{21}\left(J_{03}-2 m_{T} a^{2}\right) \Delta_{12}+J_{01} J_{23}\left(J_{01}+m_{T} a^{2}\right) \Delta_{23}\right]\right. \\
& \left.+J_{02}\left[-J_{03} J_{21}\left(2 J_{03}+5 m_{T} a^{2}\right) \Delta_{12}+2 J_{01} J_{23}\left(J_{01}+m_{T} a^{2}\right) \Delta_{23}\right]\right] \\
\Upsilon_{37}= & -a\left[m_{T} a^{2}\left[J_{03} J_{22}\left(-J_{03}+2 m_{T} a^{2}\right) \Delta_{12}+J_{02} J_{23}\left(J_{02}+m_{T} a^{2}\right) \Delta_{23}\right]\right. \\
& \left.+J_{01}\left[J_{03} J_{22}\left(2 J_{03}+5 m_{T} a^{2}\right) \Delta_{12}+2 J_{02} J_{23}\left(J_{02}+m_{T} a^{2}\right) \Delta_{23}\right]\right]
\end{aligned}
$$

Its determinant is given by $\Delta /\left(J_{03}^{5}\left(J_{01}+m_{T} a^{2}\right)^{5}\left(J_{02}+m_{T} a^{2}\right)^{5}\right)$, where

$$
\begin{aligned}
\Delta= & -a^{2} \Delta_{12}\left[J_{03}\left(J_{03}^{2} \Delta_{12}^{2}+J_{02}^{2} \Delta_{13}^{2}+J_{01}^{2} \Delta_{23}^{2}\right)\right. \\
& +m_{T} a^{2}\left(4 J_{03}^{2} \Delta_{12}^{2}+3 J_{02}^{2} \Delta_{13}^{2}+3 J_{01}^{2} \Delta_{23}^{2}+2 J_{03}\left[J_{02} \Delta_{13}^{2}+J_{01} \Delta_{23}^{2}\right]\right) \\
& \left.+m_{T}^{2} a^{4}\left(3 J_{02} \Delta_{13}^{2}+3 J_{01} \Delta_{23}^{2}+J_{03}\left[4 \Delta_{12}^{2}+\Delta_{13}^{2}+\Delta_{23}^{2}\right]\right)\right]\left[J_{03}\left(J_{03}+2 m_{T} a^{2}\right) \Delta_{12}^{2}\right. \\
& \left.+J_{02}\left(J_{02}+m_{T} a^{2}\right) \Delta_{13}^{2}+J_{01}\left(J_{01}+m_{T} a^{2}\right) \Delta_{23}^{2}\right]
\end{aligned}
$$


Hence, if $\Delta_{12} \neq 0$ or equivalently $J_{12} J_{21} \neq J_{11} J_{22}$, then the system is locally fiber configuration controllable at the equilibrium $R_{e}=I_{3}$.

\section{A.3. Proof of Lemma 10}

We first show that if $\left\{A_{1}\left(\Gamma_{e}\right), A_{2}\left(\Gamma_{e}\right), \Gamma_{e}\right\}$ are linearly independent and if $\Gamma_{e} \cdot A_{i}\left(\Gamma_{e}\right) \neq 0$ for $i=1$ or $i=2$, then the good-bad symmetric product condition is satisfied at the equilibrium. The second-degree symmetric products generated from $\{\mathscr{Y} \cup \operatorname{grad} V\}$ are

$$
\begin{gathered}
\left\langle Y_{i}^{A}: Y_{j}^{A}\right\rangle^{\sim}=m_{T} a M^{-1}(\Gamma)\left[\begin{array}{c}
\left.\left[\left(\rho_{c} \times \Gamma\right) \cdot A_{i}(\Gamma)\right] A_{j}(\Gamma)+\left[\left(\rho_{c} \times \Gamma\right) \cdot A_{j}(\Gamma)\right] A_{i}(\Gamma)\right] \\
\star
\end{array}\right] \\
i, j=1,2 \\
\left\langle\operatorname{grad} V: Y_{i}^{A}\right\rangle^{\sim}=m_{T} a_{g} M^{-1}(\Gamma)\left[\begin{array}{c}
\Gamma \times\left[\rho_{c} \times A_{i}(\Gamma)\right]+m_{T} a\left\{\left[\rho_{c} \times \Gamma\right] \cdot A_{i}(\Gamma)\right\} \tilde{M}_{11}(\Gamma)\left[\Gamma \times \rho_{c}\right] \\
+m_{T} a\left\{\left[\rho_{c} \times \Gamma\right] \cdot \tilde{M}_{11}(\Gamma)\left[\Gamma \times \rho_{c}\right]\right\} A_{i}(\Gamma) \\
\star
\end{array}\right] \\
\quad i=1,2
\end{gathered}
$$

where $\star$ denotes the terms that can be spanned by the corresponding terms in $Y_{i}^{A}$, and

$$
M^{-1}(\Gamma)=\tilde{M}(\Gamma)=\left[\begin{array}{cc}
\tilde{M}_{11}(\Gamma) & \tilde{M}_{12}(\Gamma) \\
\widetilde{M}_{21}(\Gamma) & \widetilde{M}_{22}(\Gamma)
\end{array}\right]
$$

At an equilibrium where $\rho_{c} \times \Gamma_{e}=0$, two bad symmetric products are evaluated as

$$
\left\langle Y_{i}^{A}: Y_{i}^{A}\right\rangle \sim\left(\Gamma_{e}\right)=-m_{T} a M^{-1}\left(\Gamma_{e}\right)\left[\begin{array}{l}
0 \\
\star
\end{array}\right], \quad i=1,2
$$

and three good symmetric products are evaluated as

$$
\begin{aligned}
\left\langle Y_{1}^{A}: Y_{2}^{A}\right\rangle^{\sim}\left(\Gamma_{e}\right) & =-m_{T} a M^{-1}\left(\Gamma_{e}\right)\left[\begin{array}{l}
0 \\
\star
\end{array}\right] \\
\left\langle\operatorname{grad} V: Y_{i}^{A}\right\rangle^{\sim}\left(\Gamma_{e}\right) & =m_{T} a_{g} M^{-1}\left(\Gamma_{e}\right)\left[\begin{array}{c}
\Gamma_{e} \times\left[\rho_{c} \times A_{i}\left(\Gamma_{e}\right)\right] \\
\star
\end{array}\right], \quad i=1,2
\end{aligned}
$$

Hence, these bad symmetric products are linear combinations of $Y_{1}^{A}$ and $Y_{2}^{A}$ at the equilibrium.

Consider the third-degree bad symmetric products

$$
\left\langle\left\langle Y_{i}^{A}: Y_{i}^{A}\right\rangle: \operatorname{grad} V\right\rangle, \quad\left\langle\left\langle\operatorname{grad} V: Y_{i}^{A}\right\rangle: Y_{i}^{A}\right\rangle, \quad i=1,2
$$


Note that the equilibrium condition implies that $\rho_{c}=\lambda \Gamma_{e}$ for some nonzero $\lambda$. Using this result and formula (19), one can verify that when evaluated at the equilibrium,

$$
\left\langle\left\langle Y_{i}^{A}: Y_{i}^{A}\right\rangle: \operatorname{grad} V\right\rangle \sim\left(\Gamma_{e}\right)=m_{T} a_{g} M^{-1}\left(\Gamma_{e}\right)\left[\begin{array}{c}
\Gamma_{e} \times h_{i}\left(\Gamma_{e}\right) \\
\star
\end{array}\right], \quad i=1,2
$$

for some smooth functions $h_{i}\left(\Gamma_{e}\right) \in \mathbb{R}^{3}$ (for which we do not need their explicit expressions), and

$$
\begin{aligned}
& \left\langle\left\langle\operatorname{grad} V: Y_{i}^{A}\right\rangle: Y_{i}^{A}\right\rangle \sim\left(\Gamma_{e}\right) \\
& \quad=m_{T} a_{g} M^{-1}\left(\Gamma_{e}\right)\left[\begin{array}{c}
\Gamma_{e} \times\left[\rho_{c} \times \frac{\partial A_{i}}{\partial \Gamma}\left(A_{i}\left(\Gamma_{e}\right) \times \Gamma_{e}\right)\right]+\left(\Gamma_{e} \times\left[\rho_{c} \times A_{i}\left(\Gamma_{e}\right)\right]\right) \times A_{i}\left(\Gamma_{e}\right) \\
\star
\end{array}\right]
\end{aligned}
$$

Since $\rho_{c}=\lambda \Gamma_{e}$ and

$$
\begin{aligned}
\left(\Gamma_{e} \times\left[\rho_{c} \times A_{i}\left(\Gamma_{e}\right)\right]\right) \times A_{i}\left(\Gamma_{e}\right) & =-\left(A_{i}\left(\Gamma_{e}\right) \cdot\left[\rho_{c} \times A_{i}\left(\Gamma_{e}\right)\right]\right) \Gamma_{e}+\left(A_{i}\left(\Gamma_{e}\right) \cdot \Gamma_{e}\right)\left[\rho_{c} \times A_{i}\left(\Gamma_{e}\right)\right] \\
& =\lambda\left(A_{i}\left(\Gamma_{e}\right) \cdot \Gamma_{e}\right)\left[\Gamma_{e} \times A_{i}\left(\Gamma_{e}\right)\right]
\end{aligned}
$$

we further obtain

$$
\left\langle\left\langle\operatorname{grad} V: Y_{i}^{A}\right\rangle: Y_{i}^{A}\right\rangle^{\sim}\left(\Gamma_{e}\right)=m_{T} a_{g} M^{-1}\left(\Gamma_{e}\right)\left[\begin{array}{c}
\Gamma_{e} \times l_{i}\left(\Gamma_{e}\right) \\
\star
\end{array}\right]
$$

at the equilibrium, where $l_{i}\left(\Gamma_{e}\right) \in \mathbb{R}^{3}$ are smooth functions. Because $\left\{A_{1}\left(\Gamma_{e}\right), A_{2}\left(\Gamma_{e}\right), \Gamma_{e}\right\}$ are linearly independent, so are $\left\{\Gamma_{e} \times A_{1}\left(\Gamma_{e}\right), \Gamma_{e} \times A_{2}\left(\Gamma_{e}\right), \Gamma_{e}\right\}$. Hence, these bad symmetric products can be written as linear combinations of the lower degree good symmetric products $\langle\operatorname{grad} V$ : $\left.Y_{i}^{A}\right\rangle\left(\Gamma_{e}\right), Y_{i}^{A}\left(\Gamma_{e}\right), i=1,2$, at the equilibrium.

Consider the third-degree good symmetric products evaluated at an equilibrium:

$$
\begin{aligned}
& \left\langle\left\langle Y_{i}^{A}: Y_{j}^{A}\right\rangle: Y_{k}^{A}\right\rangle^{\sim}\left(\Gamma_{e}\right)=m_{T} a M^{-1}\left(\Gamma_{e}\right) \\
& \quad \times\left[\begin{array}{c}
{\left[\left(\rho_{c} \times\left[A_{k}\left(\Gamma_{e}\right) \times \Gamma_{e}\right]\right) \cdot A_{i}\left(\Gamma_{e}\right)\right] A_{j}\left(\Gamma_{e}\right)+\left[\left(\rho_{c} \times\left[A_{k}\left(\Gamma_{e}\right) \times \Gamma_{e}\right]\right) \cdot A_{j}\left(\Gamma_{e}\right)\right] A_{i}\left(\Gamma_{e}\right)} \\
\star
\end{array}\right]
\end{aligned}
$$

where $i, j, k=1,2$. Using the identity $\rho_{c} \times\left[A_{k}\left(\Gamma_{e}\right) \times \Gamma_{e}\right]=\left[\rho_{c} \cdot A_{k}\left(\Gamma_{e}\right)\right] \Gamma_{e}-\left[\rho_{c} \cdot \Gamma_{e}\right] A_{k}\left(\Gamma_{e}\right)$, we have

$$
\left\langle\left\langle Y_{i}^{A}: Y_{i}^{A}\right\rangle: Y_{i}^{A}\right\rangle \sim\left(\Gamma_{e}\right)=m_{T} a M^{-1}\left(\Gamma_{e}\right)\left[\begin{array}{c}
\lambda\left(\left[\Gamma_{e} \cdot A_{i}\left(\Gamma_{e}\right)\right]^{2}-\left\|A_{i}\left(\Gamma_{e}\right)\right\|^{2}\right) A_{i}\left(\Gamma_{e}\right) \\
\star
\end{array}\right], \quad i=1,2
$$

It is clear that if $A_{i}\left(\Gamma_{e}\right)$ and $\Gamma_{e}$ are linearly independent, then $\left[\Gamma_{e} \cdot A_{i}\left(\Gamma_{e}\right)\right]^{2}-\left\|A_{i}\left(\Gamma_{e}\right)\right\|^{2} \neq 0$. Moreover, the term $\Gamma_{e} \times\left[\rho_{c} \times A_{i}\left(\Gamma_{e}\right)\right]$ in $\left\langle\operatorname{grad} V: Y_{i}^{A}\right\rangle\left(\Gamma_{e}\right)$ can be written as

$$
\Gamma_{e} \times\left[\rho_{c} \times A_{i}\left(\Gamma_{e}\right)\right]=\left[\Gamma_{e} \cdot A_{i}\left(\Gamma_{e}\right)\right] \rho_{c}-\left[\Gamma_{e} \cdot \rho_{c}\right] A_{i}\left(\Gamma_{e}\right)=\lambda\left(\left[\Gamma_{e} \cdot A_{i}\left(\Gamma_{e}\right)\right] \Gamma_{e}-A_{i}\left(\Gamma_{e}\right)\right)
$$


Using this result, we see that if $\left\{A_{1}\left(\Gamma_{e}\right), A_{2}\left(\Gamma_{e}\right), \Gamma_{e}\right\}$ are linearly independent, and if $\Gamma_{e}$. $A_{i}\left(\Gamma_{e}\right) \neq 0$ for $i=1$ or $i=2$, then $\left\{\Gamma_{e} \times\left[\rho_{c} \times A_{1}\left(\Gamma_{e}\right)\right], \Gamma_{e} \times\left[\rho_{c} \times A_{2}\left(\Gamma_{e}\right)\right], A_{i}\left(\Gamma_{e}\right)\right\}$ are linearly independent for $i=1$ or $i=2$. This means that any bad symmetric products of degree higher than three can be expressed as a linear combination of the lower degree good symmetric products $Y_{i}^{A},\left\langle\operatorname{grad} V: Y_{i}^{A}\right\rangle$ and $\left\langle\left\langle Y_{i}^{A}: Y_{i}^{A}\right\rangle: Y_{i}^{A}\right\rangle$ when evaluated at the equilibrium. Consequently, the good-bad symmetric product condition is satisfied.

Finally, we show that the sufficient conditions we have proved are equivalent to the conditions stated in the lemma. Since $A_{i}\left(\Gamma_{e}\right)=M_{11}^{-1}\left(\Gamma_{e}\right) M_{12}(i), i=1,2,\left\{A_{1}\left(\Gamma_{e}\right), A_{2}\left(\Gamma_{e}\right), \Gamma_{e}\right\}$ are linearly independent if and only if $\left\{M_{12}(1), M_{12}(2), M_{11}\left(\Gamma_{e}\right) \Gamma_{e}\right\}$ are linearly independent. Using the expression for $M_{11}$ and the equilibrium condition, we have $M_{11}\left(\Gamma_{e}\right) \Gamma_{e}=J \Gamma_{e}$. Moreover, it is noted that the condition $\Gamma_{e} \cdot A_{i}\left(\Gamma_{e}\right) \neq 0$ for $i=1$ or $i=2$ is equivalent to $\left(J^{-1} \Gamma_{e}\right) \cdot M_{12}(i) \neq 0$ for $i=1$ or $i=2$; the latter is further equivalent to $\left(J^{-1} \Gamma_{e}\right) \times\left[M_{12}(1) \times M_{12}(2)\right] \neq 0$. This yields equivalence of the two conditions.

\section{REFERENCES}

1. Routh EJ. Treatise on the Dynamics of a System of Rigid Bodies. MacMillan: London, 1860.

2. Chaplygin SA. On a ball's rolling on a horizontal plane (translation). Regular and Chaotic Dynamics 2002; 7(2):131-148.

3. Borisov AV, Mamaev IS. The rolling motion of a rigid body on a plane and a sphere: hierarchy dynamics. Regular and Chaotic Dynamics 2002; 7(2):177-200.

4. Cushman R. Routh's sphere. Reports on Mathematical Physics 1998; 42(1-2):47-70.

5. Fedorov YN, Kozlov VV. Various aspects of $n$-dimensional rigid body dynamics. Translations of the American Mathematical Society 1995; 168:141-171.

6. Schneider DA. Nonholonomic Euler-Poincaré equations and stability in Chaplygin's sphere. Dynamical Systems 2002; 17(2):87-130.

7. Brockett RW, Dai L. Nonholonomic kinematics and the role of elliptic function in constructive controllability. In Nonholonomic Motion Planning, Li Z, Canny JF (eds). Kluwer: Dordrecht, 1992; 1-22.

8. Jurdjevic V. The geometry of the plate-ball problem. Archive for Rational Mechanics and Analysis 1993; 124(4):305-328.

9. Mukherjee R, Minor MA, Pukrushpan JT. Motion planning for a spherical mobile robot: revisiting the classical ball-plane problem. ASME Journal of Dynamical Systems, Measurement and Control 2002; 124(4):502-511.

10. Das T, Mukherjee R, Yuksel H. Design considerations in the development of a spherical mobile robot. Proceedings of the 15th SPIE Annual International Symposium on Aerospace/Defense Sensing, Simulation, and Controls, Orlando, FL, April 2001.

11. Bloch AM. Nonholonomic Mechanics and Control. Springer: New York, 2003.

12. Bullo F. Series expansions for the evolution of mechanical control systems. SIAM Journal on Control and Optimization 2001; 40(1):166-190.

13. Lewis AD, Murray RM. Controllability of simple mechanical control systems. SIAM Journal on Control and Optimization 1997; 35(3):766-790.

14. Lewis AD. Simple mechanical control systems with constraints. IEEE Transactions on Automatic Control 2000; 45(8):1420-1436.

15. Shen J. Nonlinear control of multibody systems with symmetries via shape change. Ph.D. Thesis, Department of Aerospace Engineering, The University of Michigan, Ann Arbor, MI, 2002.

16. Shen J, Schneider DA, Bloch AM. Controllability and motion planning of multibody systems with nonholonomic constraints. Proceedings of the 42nd IEEE Conference on Decision and Control, Mauii, HI, 2003; 4369-4374.

17. Cortes J, Martinez S, Ostrowski JP, Zhang H. Simple mechanical control systems with constraints and symmetry. SIAM Journal on Control and Optimization 2002; 41(3):851-874.

18. Sussmann HJ. A general theorem on local controllability. SIAM Journal on Control and Optimization 1987; 25(5):158-194. 
19. Shen J, McClamroch NH, Bloch AM. Local equilibrium controllability of multibody systems controlled via shape change. IEEE Transactions on Automatic Control 2004; 49(4):506-520.

20. Bullo F, Zefran M. On mechanical control systems with nonholonomic constraints and symmetries. Systems and Control Letters 2001; 45(2):133-143.

21. Lewis AD. Local configuration controllability of simple mechanical control systems. Proceedings of the European Control Conference, Brussels, Belgium, 1997.

22. Sussmann HJ. Lie brackets and local controllability: a sufficient condition for scalar-input systems. SIAM Journal on Control and Optimization 1983; 21(5):686-713. 